\title{
HIGH ORDER APPROXIMATION OF PROBABILISTIC SHOCK PROFILES IN HYPERBOLIC CONSERVATION LAWS WITH UNCERTAIN INITIAL DATA *,**
}

\author{
Christoph Schwab ${ }^{1}$ and Svetlana Tokareva ${ }^{1}$
}

\begin{abstract}
We analyze the regularity of random entropy solutions to scalar hyperbolic conservation laws with random initial data. We prove regularity theorems for statistics of random entropy solutions like expectation, variance, space-time correlation functions and polynomial moments such as gPC coefficients. We show how regularity of such moments (statistical and polynomial chaos) of random entropy solutions depends on the regularity of the distribution law of the random shock location of the initial data. Sufficient conditions on the law of the initial data for moments of the random entropy solution to be piece-wise smooth functions of space and time are identified, even in cases where path-wise random entropy solutions are discontinuous almost surely. We extrapolate the results to hyperbolic systems of conservation laws in one space dimension. We then exhibit a class of stochastic Galerkin discretizations which allows to derive closed deterministic systems of hyperbolic conservation laws for the coefficients in truncated polynomial chaos expansions of the random entropy solution. Based on the regularity theory developed here, we show that depending on the smoothness of the law of the initial data, arbitrarily high convergence rates are possible for the computation of coefficients in gPC approximations of random entropy solutions for Riemann problems with random shock location by combined Stochastic Galerkin Finite Volume schemes.
\end{abstract}

Mathematics Subject Classification. 65C30, 65M08, 65M12, 65M70, 65M75.

Received September 8, 2011. Revised June 25, 2012.

Published online March 29, 2013.

\section{INTRODUCTION}

We consider the multidimensional hyperbolic system of conservation laws

$$
\frac{\partial \mathbf{U}}{\partial t}+\sum_{k=1}^{d} \frac{\partial \mathbf{F}_{k}(\mathbf{U})}{\partial x_{k}}=\mathbf{0}, x=\left(x_{1}, \ldots, x_{d}\right) \in \mathbb{R}^{d}, t>0 ;
$$

Keywords and phrases. Uncertainty quantification, hyperbolic conservation laws, probabilistic shock profile, stochastic Galerkin finite volume schemes.

* This work is performed as part of ETH interdisciplinary research grant CH1-03 10-1 and under ERC grant AdG 247277.

** The authors thank Professor S. Mishra for numerous constructive and critical remarks and stimulating discussions.

1 ETH Zürich, Seminar for Applied Mathematics, Zürich, Switzerland. christoph.schwab@sam.math.ethz.ch;

svetlana.tokareva@sam.math.ethz.ch 
with random initial data depending on $\omega \in \Omega$ :

$$
\mathbf{U}(x, 0, \omega)=\mathbf{U}_{0}(x, \omega), x \in \mathbb{R}^{d}, \omega \in \Omega .
$$

Here $\Omega$ denotes the set of all elementary events in a probability space $(\Omega, \mathcal{F}, \mathbb{P})$ on which the randomness of the initial data in (1.2) is modelled, and the random solution $\Omega \ni \omega \mapsto \mathbf{U}=\left[u_{1}, \ldots, u_{p}\right]^{\top}$ is a measurable mapping from $\mathcal{F}$ into a suitable space of vector-valued functions, defined on $\mathbb{R}^{d} \times[0, \infty)$ and taking values in a set of states $\mathbb{S} \subset \mathbb{R}^{p}$. The (deterministic) flux functions $\mathbf{F}_{k}, 1 \leqslant k \leqslant d$ in (1.1) are assumed to be known smooth functions from $\mathbb{S}$ into $\mathbb{R}^{p}$.

For smooth deterministic initial data, the unique entropy solutions of (1.1)-(1.2) generally develop discontinuities in finite time. Therefore the admissible solutions to (1.1)-(1.2) are sought in the weak sense, augmented by additional entropy criteria to ensure uniqueness and satisfy physical requirements. In the scalar multidimensional case there exists a well-posedness result for entropy solutions, however, no global well-posedness results are available for general multidimensional systems of conservation laws.

Many efficient numerical methods have been developed to approximate the entropy solutions of systems of conservation laws $[1,2]$, e.g. finite volume or discontinuous Galerkin methods. The classical assumption in designing efficient numerical methods is that the initial data $\mathbf{U}_{0}$ is known exactly. However, in many practical applications it is not always possible to obtain exact initial data due to, for example, measurement or modeling errors. In the present paper, we follow [3] and describe incomplete information in the initial data (1.2) mathematically as random fields. Such initial data are described in terms of statistical quantities of interest like the mean, variance, higher statistical moments; in some cases the distribution law of the stochastic initial data is also assumed to be known. In any of these situations one needs a mathematical formulation of (1.1)-(1.2) allowing random initial data.

A mathematical framework of random entropy solutions for scalar conservation laws has been developed in [3], to which we refer for a definition of random entropy solutions. There, existence and uniqueness of random entropy solutions to (1.1)-(1.2) has been shown for scalar hyperbolic conservation laws, also in multiple dimensions. Furthermore, the existence of the statistical quantities of the random entropy solution such as the statistical mean and $k$-point spatio-temporal correlation functions under suitable assumptions on the random initial data have been proven.

In this paper, we address the questions of regularity of the probabilistic shock profile as well as of the higher statistical and polynomial moments of the solution to the hyperbolic equation or to hyperbolic systems of equations with random initial data. It has been observed that in the case when the initial data (1.2) is assumed to be the Riemann data with random initial discontinuity position, the resulting statistical quantities (mean, variance and space-time correlation functions) are always smoother than the deterministic solution resulting from each realization of the random input (we call it path-wise solution). Moreover, the degree of smoothness depends on the smoothness of the probability density function of a given distribution. For instance, if the position of the shock is distributed uniformly on some interval, i.e. the distribution function is discontinuous, the statistical mean is nevertheless a continuous function. If, on the other hand, the location of the shock in the initial data is normally distributed so that the probability density function is infinitely differentiable, then we show that the mean random entropy solution is also an infinitely differentiable function.

Numerical methods for uncertainty quantification in hyperbolic conservation laws have been studied in e.g. $[3-7,12,13,15-17,24]$. In numerical experiments in $[3,4]$ (which were based on some form of path-wise simulation) increased smoothness of statistical quantities derived from random entropy solution has been observed numerically. The mathematical study of the regularity of statistical moments of random entropy solutions for the scalar conservation law (1.1)-(1.2) is one purpose of this paper. We present elements of a mathematical regularity theory which justifies, at least in one space dimension, the increase in space-time regularity of certain ensemble averages of the random entropy solution. Our main finding is that for a probability measure which is non-atomic (with respect to Lebesgue measure), statistics of random entropy solutions are more regular than path-wise solutions. We exhibit, as extreme cases, classes of probabilities distributions which render the statistics of the random entropy solutions smooth. We emphasize that this smoothing effect is not related to the classical 
concept of viscous shock profile: all conservation laws considered in the present paper are considered without any viscous regularization.

We present consequences of this observation for the design of effective numerical solvers for (1.1)-(1.2).

As alluded to above, the convergence order of solution approaches based on sample averages of path-wise numerical solutions are constrained by the (inevitable, in general) shocks forming in entropy solutions of (1.1). These imply only first-order convergence rates regardless of the formal order of the numerical scheme used.

In the light of our regularity theory, therefore, superior performance of numerical schemes which directly approximate statistical quantities can be anticipated. One example of such solution schemes is the Stochastic Galerkin Finite Volume Method ("sGFVM" for short) where the primary unknowns are coefficients in generalized polynomial chaos ("gPC" for short) expansions of the solution. These coefficients can be, as we show, be considerably smoother than the path-wise solution.

The outline of the present paper is as follows: in Section 2, we recapitulate basic concepts from probability and random variables taking values in function spaces. We recapitulate in particular the notion of random entropy solution from [3]. Section 3 then addresses the regularity of the mean field (or ensemble average) of random entropy solutions with Riemann-type initial data. Section 4 generalizes the result to two- and $n$-point spatio-temporal correlation functions. Section 5 establishes analogous results for gPC expansions of the random entropy solution. In Section 6, the generalizations of the results to systems are discussed and another type of random initial data is considered. Section 7 addresses the consequences of the regularity results for high order approximations of random solutions.

\section{Preliminaries}

\subsection{Probability spaces}

We introduce a probability space $(\Omega, \mathcal{F}, \mathbb{P})$, with $\Omega$ being the set of all elementary events, or space of outcomes, and $\mathcal{F}$ a $\sigma$-algebra of all possible events, equipped with a probability measure $\mathbb{P}$. Random entropy solutions are random functions taking values in a function space; to this end, let $(E, \mathcal{G}, \mathbb{G})$ denote any measurable space. Then an $E$-valued random variable is any mapping $Y: \Omega \rightarrow E$ such that the set $\{\omega \in \Omega: Y(\omega) \in A\}=\{Y \in A\} \in \mathcal{F}$ for any $A \in \mathcal{G}$, i.e. such that $Y$ is a $\mathcal{G}$-measurable mapping from $\Omega$ into $E$.

We confine ourselves to the case that $E$ is a complete metric space; then $(E, \mathcal{B}(E))$ equipped with a Borel $\sigma$-algebra $\mathcal{B}(E)$ is a measurable space. By definition, $E$-valued random variables $Y: \Omega \rightarrow E$ are $(E, \mathcal{B}(E))$ measurable. Furthermore, if $E$ is a separable Banach space with norm $\|\circ\|_{E}$ and with topological dual $E^{*}$, then $\mathcal{B}(E)$ is the smallest $\sigma$-algebra of subsets of $E$ containing all sets

$$
\{x \in E: \varphi(x)<\alpha\}, \varphi \in E^{*}, \alpha \in \mathbb{R} .
$$

Hence, if $E$ is a separable Banach space, $Y: \Omega \rightarrow E$ is an $E$-valued random variable if and only if for every $\varphi \in E^{*}, \omega \mapsto \varphi(Y(\omega)) \in \mathbb{R}$ is an $\mathbb{R}$-valued random variable. Moreover, there hold the following results on existence and uniqueness [3].

Lemma 2.1. Let $E$ be a separable Banach space and let $Y: \Omega \rightarrow E$ be an E-valued random variable on $(\Omega, \mathcal{F})$. Then the mapping $\omega \mapsto\|Y(\omega)\|_{E} \in \mathbb{R}$ is measurable.

Definition 2.2. The random variable $Y: \Omega \rightarrow E$ is called Bochner integrable if, for any probability measure $\mathbb{P}$ on the measurable space $(\Omega, \mathcal{F})$, it holds

$$
\int_{\Omega}\|Y(\omega)\|_{E} \mathbb{P}(\mathrm{d} \omega)<\infty .
$$

Definition 2.3. A probability measure is any $\sigma$-additive set function $\mathbb{P}: \Omega \rightarrow[0,1]$ such that $\mathbb{P}(\Omega)=1$. The measure space $(\Omega, \mathcal{F}, \mathbb{P})$ is called probability space.

We shall always assume that the probability space is complete. 
Definition 2.4. If $Y:(\Omega, \mathcal{F}) \rightarrow(E, \mathcal{G})$ is a random variable, then

$$
\mathcal{L}(Y)(A)=\mathbb{P}(\{\omega \in \Omega: Y(\omega) \in A\}), \forall A \in \mathcal{G}
$$

denotes the law of $Y$ under $\mathbb{P}$. The image measure $\mu_{Y}=\mathcal{L}(Y)$ on $(E, \mathcal{G})$ is called law or distribution of $Y$.

Definition 2.5. A random variable taking values in $E$ is called simple if it can take only finitely many values, i.e. if it has the explicit form

$$
Y=\sum_{i=1}^{N} y_{i} \chi_{A_{i}}, A_{i} \in \mathcal{F}, x_{i} \in E, N<\infty,
$$

where $\chi_{A}$ is the indicator function of $A \in \mathcal{F}$.

For a simple $E$-valued random variable $Y$ and for any $B \in \mathcal{F}$ we set

$$
\int_{B} Y(\omega) \mathbb{P}(\mathrm{d} \omega)=\int_{B} Y d \mathbb{P}=\sum_{i=1}^{N} x_{i} \mathbb{P}\left(A_{i} \cap B\right) .
$$

For such $Y(\omega)$ and all $B \in \mathcal{F}$ holds

$$
\left\|\int_{B} Y(\omega) \mathbb{P}(\mathrm{d} \omega)\right\|_{E} \leqslant \int_{B}\|Y(\omega)\|_{E} \mathbb{P}(\mathrm{d} \omega) .
$$

For any random variable $Y: \Omega \rightarrow E$ which is Bochner integrable, there exists a sequence $\left\{Y_{m}\right\}_{m \in \mathbb{N}}$ of simple random variables such that, for all $\omega \in \Omega,\left\|Y(\omega)-Y_{m}(\omega)\right\|_{E} \rightarrow 0$ as $m \rightarrow \infty$. Therefore (2.1) and (2.2) can be extended to any $E$-valued random variable. We denote the expectation of $Y$ by

$$
\mathbb{E}[Y]=\int_{\Omega} Y(\omega) \mathbb{P}(\mathrm{d} \omega)=\lim _{m \rightarrow \infty} \int_{\Omega} Y_{m}(\omega) \mathbb{P}(\mathrm{d} \omega) \in E .
$$

Denote by $L^{p}(\Omega, \mathcal{F}, \mathbb{P} ; E)$ for $1 \leqslant p \leqslant \infty$ the Bochner space of all $p$-summable, $E$-valued random variables $Y$ and equip it with the norm

$$
\|Y\|_{L^{p}(\Omega ; E)}=\left(\mathbb{E}\left[\|Y\|_{E}^{p}\right]\right)^{1 / p}=\left(\int_{\Omega}\|Y(\omega)\|_{E}^{p} \mathbb{P}(\mathrm{d} \omega)\right)^{1 / p} .
$$

For $p=\infty$ we can denote by $L^{\infty}(\Omega, \mathcal{F}, \mathbb{P} ; E)$ the set of all $E$-valued random variables which are essentially bounded and equip this space with the norm

$$
\|Y\|_{L^{\infty}(\Omega ; E)}=\operatorname{ess} \sup _{\omega \in \Omega}\|Y(\omega)\|_{E} .
$$

\subsection{Scalar conservation laws with random initial data}

Consider the Cauchy problem for the scalar hyperbolic conservation law

$$
\frac{\partial u}{\partial t}+\sum_{j=1}^{d} \frac{\partial f_{j}(u)}{\partial x_{j}}=0, x=\left(x_{1}, \ldots, x_{d}\right) \in \mathbb{R}^{d}, t>0
$$

and random initial condition

$$
u(x, 0, \omega)=u_{0}(x, \omega), x \in \mathbb{R}^{d}, \omega \in \Omega
$$


Assume we have as $u_{0}$ an $L^{1}\left(\mathbb{R}^{d}\right)$-valued random variable

$$
u_{0}:(\Omega, \mathcal{F}) \rightarrow\left(L^{1}\left(\mathbb{R}^{d}\right), \mathcal{B}\left(L^{1}\left(\mathbb{R}^{d}\right)\right)\right)
$$

and that

$$
u_{0}(\cdot, \omega) \in L^{\infty}\left(\mathbb{R}^{d}\right) \cap B V\left(\mathbb{R}^{d}\right) \mathbb{P}-\text { a.s. }
$$

Since $L^{1}\left(\mathbb{R}^{d}\right)$ is separable, the mapping $u_{0}$ is well defined and we may impose for $k \in \mathbb{N}$ the $k$-th moment condition

$$
\left\|u_{0}\right\|_{L^{k}\left(\Omega ; L^{1}\left(\mathbb{R}^{d}\right)\right)}<\infty .
$$

Definition 2.6 (Random entropy solution). A random field

$$
u: \omega \in \Omega \mapsto u(x, t, \omega) \in C^{0}\left([0, T] ;\left(L^{1} \cap L^{\infty}\right)\left(\mathbb{R}^{d}\right)\right)
$$

is said to be a random entropy solution if it satisfies $\mathbb{P}$-a.s. the following:

(i) Weak solution: for $\mathbb{P}$-a.e. $\omega \in \Omega, u$ satisfies the following integral identity,

$$
\int_{0}^{\infty} \int_{\mathbb{R}^{d}}\left(u(x, t, \omega) \frac{\partial \varphi(x, t)}{\partial t}+\sum_{j=1}^{d} f_{j}(u(x, t, \omega)) \frac{\partial \varphi(x, t)}{\partial x_{j}}\right) \mathrm{d} x \mathrm{~d} t+\int_{\mathbb{R}^{d}} u_{0}(x, \omega) \varphi(x, 0) \mathrm{d} x=0,
$$

for all test functions $\varphi \in C_{0}^{1}\left(\mathbb{R}^{d} \times[0, \infty)\right)$.

(ii) Entropy condition: for any entropy-entropy flux pair, i.e. smooth functions $\eta$ and $Q_{j}$ with $j=1, \ldots, d$ such that $\eta$ is convex and $Q_{j}^{\prime}=\eta^{\prime} f_{j}^{\prime}$ for all $j$, and for $\mathbb{P}$-a.e. $\omega \in \Omega, u$ satisfies the following integral inequality:

$$
\int_{0}^{\infty} \int_{\mathbb{R}^{d}}\left(\eta(u(x, t, \omega)) \frac{\partial \varphi(x, t)}{\partial t}+\sum_{j=1}^{d} Q_{j}(u(x, t, \omega)) \frac{\partial \varphi(x, t)}{\partial x_{j}}\right) \mathrm{d} x \mathrm{~d} t \geqslant 0
$$

for all test functions $\varphi \in C_{0}^{1}\left(\mathbb{R}^{d} \times(0, \infty)\right), \varphi \geqslant 0$.

The following well-posedness theorem for entropy solutions holds (see [3]).

Theorem 2.7. Consider the scalar conservation law (2.3)-(2.4) with random initial data $u_{0}: \Omega \rightarrow L^{1}\left(\mathbb{R}^{d}\right)$ satisfying (2.5)-(2.6) and the $k$-th moment condition (2.7) for some $k \in \mathbb{N}$, and let $\mathbb{S}: u_{0} \mapsto u=\mathbb{S}(t) u_{0}$ denote the (nonlinear) data-to-solution operator.

Then there exists a unique random entropy solution $u: \omega \in \Omega \mapsto C_{b}\left([0, T] ; L^{1}\left(\mathbb{R}^{d}\right)\right)$ given by

$$
u(\cdot, t, \omega)=\mathbb{S}(t) u_{0}(\cdot, \omega), t>0, \omega \in \Omega
$$

such that for every $k \geqslant 1$ and every $0 \leqslant t \leqslant T<\infty$ holds $\mathbb{P}$-a.s.

$$
\begin{aligned}
\|u\|_{L^{k}\left(\Omega ; C\left(0, T ; L^{1}\left(\mathbb{R}^{d}\right)\right)\right)} & \leqslant\left\|u_{0}\right\|_{L^{k}\left(\Omega ; L^{1}\left(\mathbb{R}^{d}\right)\right)}, \\
\left\|S(t) u_{0}(\cdot, \omega)\right\|_{\left(L^{1} \cap L^{\infty}\right)\left(\mathbb{R}^{d}\right)} & \leqslant\left\|u_{0}(\cdot, \omega)\right\|_{\left(L^{1} \cap L^{\infty}\right)\left(\mathbb{R}^{d}\right)}
\end{aligned}
$$

and such that we have

$$
T V\left(S(t) u_{0}(\cdot, \omega)\right) \leqslant T V\left(u_{0}(\cdot, \omega)\right) \mathbb{P}-\text { a.s. }
$$


For $u \in L^{k}(\Omega ; E)$ consider the $k$-fold tensor product and the corresponding tensor-product space:

$$
u^{(k)}=\underbrace{u \otimes \cdots \otimes u}_{k \text { times }} \in L^{1}\left(\Omega ; E^{(k)}\right), \quad E^{(k)}=\underbrace{E \otimes \cdots \otimes E}_{k \text { times }} .
$$

For any cross-norm $\|\circ\|_{E^{(k)}}$ on the tensor-product space $E^{(k)}$ we have

$$
\left\|u_{1} \otimes \cdots \otimes u_{k}\right\|_{E^{(k)}}=\left\|u_{1}\right\|_{E} \cdots\left\|u_{k}\right\|_{E} .
$$

Therefore

$$
\left\|u^{(k)}\right\|_{L^{1}\left(\Omega ; E^{(k)}\right)}=\int_{\Omega}\|u(\cdot, \omega)\|_{E}^{k} \mathbb{P}(\mathrm{d} \omega)=\|u\|_{L^{k}(\Omega ; E)}^{k}<\infty,
$$

which implies $u^{(k)} \in L^{1}\left(\Omega ; E^{(k)}\right)$ and the k-point spatio-temporal correlation function

$$
\mathcal{M}^{k}[u]=\mathbb{E}\left[u^{(k)}\right] \in E^{(k)}
$$

is well-defined for $u \in L^{k}(\Omega ; E)$. With these assumptions, the following theorem was shown in [3].

Theorem 2.8. Consider the scalar conservation law (2.3)-(2.4) with random initial data $u_{0}: \Omega \rightarrow L^{1}\left(\mathbb{R}^{d}\right)$ satisfying (2.6). Assume further that for some $k \in \mathbb{N}$ and some real number $r \geqslant 1$

$$
u_{0} \in L^{r k}\left(\Omega ; L^{1}\left(\mathbb{R}^{d}\right)\right) .
$$

Then for every $0<T<\infty$ and every $0<t_{1}, t_{2}, \ldots, t_{k} \leqslant T<\infty$ the tensor product

$$
u\left(x_{1}, t_{1}, \omega\right) \otimes \cdots \otimes u\left(x_{k}, t_{k}, \omega\right)
$$

is well-defined as an element of $L^{r}\left(\Omega ; L^{1}\left(\mathbb{R}^{k d}\right)\right)$. In particular, the $k$-point space-time correlation function

$$
\mathcal{M}^{k}[u]=\mathbb{E}\left[u\left(\cdot, t_{1}, \omega\right) \otimes \cdots \otimes u\left(\cdot, t_{k}, \omega\right)\right]
$$

is well-defined for any choice of $t_{j}$ as an element of $L^{1}\left(\mathbb{R}^{k d}\right)$ and it satisfies

$$
\left\|\mathcal{M}^{k}[u]\left(t_{1}, \ldots, t_{k}\right)\right\|_{L^{1}\left(\mathbb{R}^{d}\right)^{(k)}} \leqslant\left\|u_{0}\right\|_{L^{k}\left(\Omega, L^{1}\left(\mathbb{R}^{d}\right)\right)}^{k} .
$$

Corollary 2.9. The expecation and variance of the solution are well-defined if $r k \geqslant 2$ in Theorem 2.8 since

$$
\mathbb{E}[u]=\mathcal{M}^{1}[u] \in L^{1}\left(\mathbb{R}^{d}\right), \quad \mathbb{V}[u]=\mathbb{E}\left[(u-\mathbb{E}[u])^{2}\right] \in L^{1}\left(\mathbb{R}^{d}\right) .
$$

In the following, we restrict ourselves to $d=1$ and consider the scalar conservation law

$$
\begin{aligned}
& \frac{\partial u}{\partial t}+\frac{\partial f(u)}{\partial x}=0, x \in \mathbb{R}, t>0 \\
& u(x, 0, \omega)=u_{0}(x, \omega), \omega \in \Omega
\end{aligned}
$$

with the flux function $f \in C^{1}(\mathbb{R}), f: \mathbb{R} \rightarrow \mathbb{R}$. To analyze the regularity of the random entropy solution to (2.10)-(2.11) we make the following additional assumption on the structure of the random field $u_{0}$.

Lemma 2.10. Assume there exists a random variable $Y(\omega)$ taking values in $\mathbb{R}$ and a deterministic function $\tilde{u}_{0}(x, y)$ such that in $(2.11)$,

$$
u_{0}(x, \omega)=\left.\tilde{u}_{0}(x, y)\right|_{y=Y(\omega)} .
$$

Then for all $t>0$ the following equality for the exact random entropy solution to (2.10)-(2.11) holds:

$$
u(x, t, \omega)=\left.\tilde{u}(x, t, y)\right|_{y=Y(\omega)} .
$$


We parametrize the space of outcomes by introducing a real-valued random variable $y=Y(\omega): \Omega \ni \omega \mapsto y=$ $Y(\omega) \in \mathbb{R}$. Then the initial condition (2.11) as well as the solution of the system (2.10) can be represented as functions dependent on this stochastic variable $y$ :

$$
u_{0}=\left.\tilde{u}_{0}(x, y)\right|_{y=Y(\omega)}, u=\left.\tilde{u}(x, t, y)\right|_{y=Y(\omega)} .
$$

In the following we shall abuse the notation by omitting the tilde and writing $u$ instead of $\tilde{u}$.

The scalar conservation law (2.10)-(2.11) in parametrized form will then become

$$
\begin{aligned}
& \frac{\partial u}{\partial t}+\frac{\partial f(u)}{\partial x}=0, x \in \mathbb{R}, t>0 \\
& u(x, 0, y)=u_{0}(x, y), y \in \mathbb{R}
\end{aligned}
$$

where $u=u(x, t, y)$ is the unknown solution.

We assume throughout that the probability measure $\mathbb{P}$ is non-atomic and absolutely continuous with respect to Lebesgue measure $\lambda$ on $\mathbb{R}$. By the Radon-Nikodym theorem there exists an essentially bounded probability density function, i.e.

$$
\rho(y)=d \mathbb{P}(\omega) / \mathrm{d} y \in\left(L^{1} \cap L^{\infty}\right)(\mathbb{R}) \quad \text { such that } \quad \mathbb{P}(\mathbb{R})=\int_{\mathbb{R}} \rho(y) \mathrm{d} y=1 .
$$

We shall use the cumulative distribution function defined as

$$
P(x)=\mathbb{P}(\{y \leqslant x\})=\int_{-\infty}^{x} \rho(y) \mathrm{d} y .
$$

We are interested in the statistics of the random entropy solution such as the mean, variance, statistical and polynomial moments. The latter are of particular significance for the numerical analysis of gPC based discretizations. The expectation (or mean solution value) is defined by formula

$$
\mathbb{E}[u](x, t)=\int_{\Omega} u(x, t, \omega) \mathbb{P}(\mathrm{d} \omega)=\int_{\mathbb{R}} u(x, t, y) \rho(y) \mathrm{d} y .
$$

The variance of the random solution is formally defined by

$$
\mathbb{V}[u]=\mathbb{E}\left[(u-\mathbb{E}[u])^{2}\right]=\mathbb{E}\left[u^{2}\right]-(\mathbb{E}[u])^{2} .
$$

Assume that the initial data $u_{0}(x, y)$ is such that the solution $u(x, t, y)$ for each particular realization of $y$ (path-wise solution) is a shock wave, then in one-dimensional case it can be described by means of the Heaviside function

$$
H(x)= \begin{cases}1, & \text { if } x>0 \\ 0, & \text { if } x<0\end{cases}
$$

We shall often write it as $u(x, t, y) \sim H(x-S t-y)$, meaning that the exact solution of $(2.14)-(2.15)$ equals the Heaviside function, accurate to multiplication or addition of some constant, which apparently does not influence the regularity.

Definition 2.11. Given a random variable $Y(\omega)$ with law $d \mathbb{P}(\omega)=\rho d \lambda$ on $\mathbb{R}$, we define the probabilistic shock profile of the scalar conservation law (2.10) with random initial data (2.11) as the parametric deterministic family of random entropy solutions $u(x, t, y)$ generated by all particular realizations of $Y(\omega)$. 


\section{Regularity of THE PROBABILISTIC SHOCK PROFILE FOR ONE-DIMENSIONAL SCALAR EQUATION}

Consider the scalar hyperbolic equation in conservative form

$$
\begin{gathered}
\frac{\partial u}{\partial t}+\frac{\partial f(u)}{\partial x}=0, x \in \mathbb{R}, t>0 ; \\
u(x, 0, \omega)=u_{0}(x, \omega), \omega \in \Omega .
\end{gathered}
$$

The solution to equation (3.1) with initial condition (3.2) is generally defined by the following implicit relation:

$$
u(x, t, \omega)=u_{0}\left(\tilde{x}_{0}, \omega\right), x=\tilde{x}_{0}+f^{\prime}\left(u_{0}\left(\tilde{x}_{0}, \omega\right)\right) t .
$$

Consider the Riemann initial data generating $\mathbb{P}$-a.s. path-wise discontinuous solutions to (3.1)-(3.2):

$$
u_{0}(x, \omega)= \begin{cases}u_{L}, & \text { if } x<x_{0}+Y(\omega) ; \\ u_{R}, & \text { if } x>x_{0}+Y(\omega),\end{cases}
$$

with $u_{R}<u_{L}$. We introduce the stochastic variable $y=x_{0}+Y(\omega) \in \mathbb{R}$ and use it to derive the exact path-wise solution to equation (3.1) with Riemann initial data (3.4), which has the form of a shock wave:

$$
u(x, t, y)=u_{L}+\left(u_{R}-u_{L}\right) H(x-S t-y),
$$

where $S=\left(f\left(u_{R}\right)-f\left(u_{L}\right)\right) /\left(u_{R}-u_{L}\right)$ is the shock speed.

Let $y$ be distributed with the probability density function $\rho(y)$. Then the expectation of the probabilistic shock profile of the random entropy solution is defined as the expected solution value, i.e.

$$
\mathbb{E}[u]=\int_{-\infty}^{\infty} u(x, t, y) \rho(y) \mathrm{d} y=u_{L}+\left(u_{R}-u_{L}\right) \int_{-\infty}^{\infty} H(x-S t-y) \rho(y) \mathrm{d} y .
$$

In the following, we need the modified Heaviside function

$$
\bar{H}\left(y-y_{0}\right)= \begin{cases}1, & y<y_{0} \\ 0, & y>y_{0}\end{cases}
$$

with $y_{0}=x-S t$. Making use of the property

$$
\int \bar{H}\left(y-y_{0}\right) \varphi^{\prime}(y) \mathrm{d} y=\int \delta\left(y-y_{0}\right) \varphi(y) \mathrm{d} y,
$$

resulting in $\bar{H}^{\prime}\left(y-y_{0}\right)=-\delta\left(y-y_{0}\right)$, where $\varphi \in C_{c}^{\infty}(\mathbb{R})$ is a test function and $\delta(y)$ is the Dirac delta-function such that

$$
\int_{-\infty}^{\infty} \varphi(y) \delta\left(y-y_{0}\right) \mathrm{d} y=\varphi\left(y_{0}\right)
$$

and using the (cumulative) distribution function (2.16) we get the following expression for the mean:

$$
\begin{aligned}
\mathbb{E}[u]= & u_{L}+\left(u_{R}-u_{L}\right) \int_{-\infty}^{\infty} \rho(y) \bar{H}\left(y-y_{0}\right) \mathrm{d} y=u_{L}+\left(u_{R}-u_{L}\right)\left[\left.\bar{H}\left(y-y_{0}\right) P(y)\right|_{-\infty} ^{\infty}\right. \\
& \left.+\int_{-\infty}^{\infty} P(y) \delta\left(y-y_{0}\right) \mathrm{d} y\right]=u_{L}+0+\left(u_{R}-u_{L}\right) P\left(y_{0}\right)=u_{L}+\left(u_{R}-u_{L}\right) P(x-S t) .
\end{aligned}
$$


Therefore, the regularity of the probabilistic shock profile is entirely defined by the properties of the cumulative distribution function $P(x-S t)$. We next investigate the properties of $P(x)$ for $\rho(x)$ belonging to different functional spaces and formulate the corresponding regularity theorems.

Theorem 3.1. Let $\rho \in C^{k}(\mathbb{R})$. Then $\mathbb{E}[u] \in C^{k+1}(\mathbb{R})$ for any $t>0$.

Proof. From (2.16), $P^{\prime}(x)=\rho(x) \in C^{k}(\mathbb{R})$. Hence, $P \in C^{k+1}(\mathbb{R})$ and from (3.7) we have $\mathbb{E}[u] \in C^{k+1}(\mathbb{R})$.

Remark 3.2. Note that the integrability of $\rho$ over $\mathbb{R}$ implies that it is at least piecewise-continuous function, that is, $\rho \in C^{-1}(\mathbb{R})$. Hence, by Theorem 3.1, for $t>0$ the expectation of the random entropy solutions is a continuous functions of $x$ on the whole domain $\mathbb{R}$, i.e. $\mathbb{E}[u] \in C^{0}(\mathbb{R})$.

Theorem 3.3. Let $\rho \in C^{\infty}(\mathbb{R})$. Then $\mathbb{E}[u] \in C^{\infty}(\mathbb{R})$ for any $t>0$.

Proof. The space $C^{\infty}(\mathbb{R})$ of infinitely differentiable functions is by our definition

$$
C^{\infty}(\mathbb{R})=\bigcap_{k \geqslant 0} C^{k}(\mathbb{R})
$$

For any $k \geqslant 0, \rho \in C^{k}(\mathbb{R}) \Rightarrow P \in C^{k+1}(\mathbb{R})$. Hence, by Theorem 3.1,

$$
\rho \in \bigcap_{k \geqslant 0} C^{k}(\mathbb{R}) \Rightarrow P \in \bigcap_{k \geqslant 0} C^{k+1}(\mathbb{R})=C^{\infty}(\mathbb{R}) .
$$

Theorem 3.4. Let $\rho \in L^{\infty}(\mathbb{R})$. Then $\mathbb{E}[u] \in W^{1, \infty}(\mathbb{R})$ for any $t>0$.

Proof. By the assumptions in the theorem, $\rho \in L^{\infty}(\mathbb{R})$, that is,

$$
\exists M>0, M<\infty:|\rho(y)| \leqslant M \text { a.e } y \in \mathbb{R} .
$$

Hence, the weak derivative of the distribution function is bounded:

$$
P^{\prime}(x)=\rho(x) \Rightarrow\left|P^{\prime}(x)\right| \leqslant M \text { a.e. } x \in \mathbb{R} .
$$

The function $P(x)$ itself is bounded: $|P(x)| \leqslant 1$ a.e. $x \in \mathbb{R}$. This implies

$$
P \in W^{1, \infty}(\mathbb{R})=\left\{f \in L^{\infty}(\mathbb{R}): f^{\prime} \in L^{\infty}(\mathbb{R})\right\} .
$$

We recall the space $C^{k, \alpha}(\mathbb{R})$ of functions whose $k$-th derivative is Hölder-continuous with exponent $\alpha$, i.e.

$$
f \in C^{k, \alpha}(\mathbb{R}) \Leftrightarrow f^{(k)} \in C^{0, \alpha}(\mathbb{R}) \Leftrightarrow \exists M>0, M<\infty:\left|f^{(k)}(x)-f^{(k)}(y)\right| \leqslant M|x-y|^{\alpha}, \forall x, y \in \mathbb{R} .
$$

Theorem 3.5. Let $\rho \in C^{k, \alpha}(\mathbb{R})$. Then $\mathbb{E}[u] \in C^{k+1, \alpha}(\mathbb{R})$ for any $t>0$.

Proof. We have $P^{\prime}=\rho \in C^{k, \alpha}(\mathbb{R}) \Leftrightarrow \rho^{(k)}=P^{(k+1)} \in C^{0, \alpha}(\mathbb{R}) \Rightarrow P \in C^{k+1, \alpha}(\mathbb{R})$. Hence, $\mathbb{E}[u] \in C^{k+1, \alpha}(\mathbb{R})$.

We next formulate and prove several results on the regularity of the probabilistic shock profiles in case of the piece-wise differentiable probability density functions.

Definition 3.6. The set $\mathcal{P}=\left\{K_{j}\right\}_{j=1}^{J}, J<\infty$ is called finite partition of the domain $D \subset \mathbb{R}$ if

$$
\text { 1) } K_{j} \text { are open, 2) } K_{i} \cap K_{j}=\emptyset \text { if } i \neq j \text {, 3) } D=\overline{\bigcup_{j} K_{j}} \text {. }
$$


Definition 3.7. A function $f$ is called piecewise $k$ times continuously differentiable in $D \subset \mathbb{R}$ with respect to the finite partition $\mathcal{P}$, i.e. $f \in C_{p w}^{k}(D, \mathcal{P})$, if $\forall K \in \mathcal{P}: f \in C^{k}(\bar{K})$.

The Hölder space $C_{p w}^{k, \alpha}(\mathbb{R}, \mathcal{P})$ is the piece-wise analogue of the Hölder space $C^{k, \alpha}(\mathbb{R})$ :

$$
\begin{aligned}
& f \in C_{p w}^{k, \alpha}(\mathbb{R}, \mathcal{P}) \Leftrightarrow \forall K \in \mathcal{P} f \in C^{k, \alpha}(\bar{K}) \Leftrightarrow f^{(k)} \in C_{p w}^{0, \alpha}(\bar{K}) \Leftrightarrow \\
& \exists M>0, M<\infty:\left|f^{(k)}(x)-f^{(k)}(y)\right| \leqslant M|x-y|^{\alpha}, \forall x, y \in \bar{K} .
\end{aligned}
$$

In the following, we shall omit explicit reference to the partition $\mathcal{P}$ when it is clear from the context. We shall further denote the partition of $\mathbb{R}$ induced by the probability density function $\rho$ as $\mathcal{P}_{\rho}$.

Theorem 3.8. Let $\rho \in C_{p w}^{k}\left(\mathbb{R}, \mathcal{P}_{\rho}\right)$. Then $\mathbb{E}[u] \in C_{p w}^{k+1}\left(\mathbb{R}, \mathcal{P}_{t}\right) \cap C^{0}(\mathbb{R})$ for any $t>0$.

Proof. Assume that the probability density function is piece-wise $k$ times continuously differentiable with respect to some partition $\mathcal{P}_{\rho}$, that is, $\rho \in C_{p w}^{k}\left(\mathbb{R}, \mathcal{P}_{\rho}\right)$, where $\mathcal{P}_{\rho}=\left\{K_{j}\right\}_{j=1}^{J}$ and $K_{j}=\left(y_{j}, y_{j+1}\right)$ is an open interval. Then the density $\rho$ can be represented as finite sum of compactly supported functions $\rho_{j}$ :

$$
\rho(y)=\sum_{j=1}^{J} \rho_{j}(y),
$$

where $\rho_{j}(y)$ is compactly supported on $K_{j}$ and $\rho_{j} \in C^{k}\left(\overline{K_{j}}\right)$. Then the expectation will be written as

$$
\begin{aligned}
\mathbb{E}[u] & =\int_{-\infty}^{\infty} u(x, t, y) \rho(y) \mathrm{d} y=u_{L}+\left(u_{R}-u_{L}\right) \int_{-\infty}^{\infty} \bar{H}\left(y-y_{0}\right) \rho(y) \mathrm{d} y \\
& =u_{L}+\left(u_{R}-u_{L}\right) \int_{-\infty}^{\infty} \bar{H}\left(y-y_{0}\right) \sum_{j=1}^{J} \rho_{j}(y)=u_{L}+\left(u_{R}-u_{L}\right) \sum_{j=1}^{J} \int_{-\infty}^{\infty} \bar{H}\left(y-y_{0}\right) \rho_{j}(y) \mathrm{d} y \\
& =u_{L}+\left(u_{R}-u_{L}\right) \sum_{j=1}^{J}\left[\left.\bar{H}\left(y-y_{0}\right) P_{j}(y)\right|_{-\infty} ^{\infty}+\int_{-\infty}^{\infty} P_{j}(y) \delta\left(y-y_{0}\right) \mathrm{d} y\right] \\
& =u_{L}+0+\left(u_{R}-u_{L}\right) \sum_{j=1}^{J} P_{j}\left(y_{0}\right)=u_{L}+\left(u_{R}-u_{L}\right) \sum_{j=1}^{J} P_{j}(x-S t)=u_{L}+\left(u_{R}-u_{L}\right) P(x-S t),
\end{aligned}
$$

where $y_{0}=x-S t$ and

$$
P_{j}(x)=\int_{-\infty}^{x} \rho_{j}(\xi) \mathrm{d} \xi
$$

is the $j$-th distribution function of the form (2.16). Each $P_{j}(x) \in C^{k+1}\left(\overline{K_{j}}\right)$ provided $\rho_{j} \in C^{k}\left(\overline{K_{j}}\right)$ (Thm. 3.1), hence, by Definition $3.7, \mathbb{E}[u] \in C_{p w}^{k+1}\left(\mathbb{R}, \mathcal{P}_{t}\right)$, where

$$
\mathcal{P}_{t}=\left\{T_{S}^{-1} K_{j}\right\}_{j=1}^{J}=\left\{\left(y_{j}+S t, y_{j+1}+S t\right)\right\}_{j=1}^{J},
$$

with $T_{S}$ denoting the shifting operator and $T_{S}^{-1}$ its inverse:

$$
T_{S} \varphi(x, t)=\varphi(x-S t), T_{S}^{-1} \varphi(x, t)=\varphi(x+S t) .
$$

Theorem 3.9. Let $\rho \in C_{p w}^{\infty}\left(\mathbb{R}, \mathcal{P}_{\rho}\right)$. Then $\mathbb{E}[u] \in C_{p w}^{\infty}\left(\mathbb{R}, \mathcal{P}_{t}\right) \cap C^{0}(\mathbb{R})$ for any $t>0$. 
Proof. To prove the theorem it is sufficient to note that $C_{p w}^{\infty}\left(\mathbb{R}, \mathcal{P}_{\rho}\right)=\bigcap_{k \geqslant 0} C_{p w}^{k}\left(\mathbb{R}, \mathcal{P}_{\rho}\right)$. Therefore, by Theorem 3.1,

$$
\rho \in \bigcap_{k \geqslant 0} C_{p w}^{k}\left(\mathbb{R}, \mathcal{P}_{\rho}\right) \Rightarrow P \in \bigcap_{k \geqslant 0} C_{p w}^{k+1}\left(\mathbb{R}, \mathcal{P}_{t}\right)=C_{p w}^{\infty}\left(\mathbb{R}, \mathcal{P}_{t}\right)
$$

and $\mathbb{E}[u] \in C_{p w}^{\infty}\left(\mathbb{R}, \mathcal{P}_{t}\right)$.

Theorem 3.10. Let $\rho \in C_{p w}^{k, \alpha}\left(\mathbb{R}, \mathcal{P}_{\rho}\right)$. Then $\mathbb{E}[u] \in C_{p w}^{k+1, \alpha}\left(\mathbb{R}, \mathcal{P}_{t}\right) \cap C^{0}(\mathbb{R})$ for any $t>0$.

Proof. Rewrite the density $\rho$ as finite sum of compactly supported functions $\rho_{j}$ :

$$
\rho(y)=\sum_{j=1}^{J} \rho_{j}(y)
$$

where $\rho_{j}(y)$ is compactly supported on $K_{j}=\left(y_{j}, y_{j+1}\right), j=1, \ldots, J$ (see the proof of Thm. 3.5) and $\rho_{j} \in$ $C^{k, \alpha}\left(\overline{K_{j}}\right)$. The distribution function is

$$
P(x)=\int_{-\infty}^{x} \sum_{j=1}^{J} \rho_{j}(y) \mathrm{d} y=\sum_{j=1}^{J} \int_{-\infty}^{x} \rho_{j}(y) \mathrm{d} y=\sum_{j=1}^{J} P_{j}(x),
$$

where each $P_{j} \in C^{k+1, \alpha}\left(\overline{K_{j}}\right)$ by Theorem 3.5. Hence, $\mathbb{E}[u] \in C_{p w}^{k+1, \alpha}\left(\mathbb{R}, \mathcal{P}_{t}\right)$, where

$$
\mathcal{P}_{t}=\left\{T_{S}^{-1} K_{j}\right\}_{j=1}^{J}=\left\{\left(y_{j}+S t, y_{j+1}+S t\right)\right\}_{j=1}^{J} .
$$

\section{Example 3.11 (Uniform distribution).}

Consider the Riemann problem for the Burgers equation

$$
\begin{gathered}
\frac{\partial u}{\partial t}+\frac{\partial}{\partial x}\left(\frac{1}{2} u^{2}\right)=0, x \in \mathbb{R}, t>0 ; \\
u(x, 0, \omega)=u_{0}(\omega)=\left\{\begin{array}{l}
u_{L}, x<x_{0}+c(2 Y(\omega)-1) ; \\
u_{R}, x>x_{0}+c(2 Y(\omega)-1),
\end{array}\right.
\end{gathered}
$$

where the position of the initial discontinuity depends on the random variable $Y(\omega)$ and $x_{0}$ and $c$ are constants. We assume $Y(\omega) \sim \mathcal{U}[0,1]$ (uniform distribution on $[0,1]$ ) and define the initial shock location as

$$
y=x_{0}+c(2 Y(\omega)-1) .
$$

Such choice implies $y \sim \mathcal{U}\left[x_{0}-c, x_{0}+c\right]$. Hence, we may represent the random initial condition (3.9) in the parametric, deterministic form

$$
u(x, 0, \omega)=u_{0}(\omega)=\left\{\begin{array}{l}
u_{L}, x<y \\
u_{R}, x>y,
\end{array}\right.
$$

If $Y(\omega) \sim \mathcal{U}[0,1]$ then its probability density function is

$$
\rho(Y(\omega))= \begin{cases}1, & Y(\omega) \in[0,1] \\ 0, & \text { otherwise }\end{cases}
$$

and its distribution function

$$
P(x)=\int_{0}^{1} \rho(Y(\omega)) \mathrm{d} y(\omega)=\left\{\begin{array}{l}
0, x<0 \\
x, x \in[0,1] \\
1, x>1
\end{array}\right.
$$



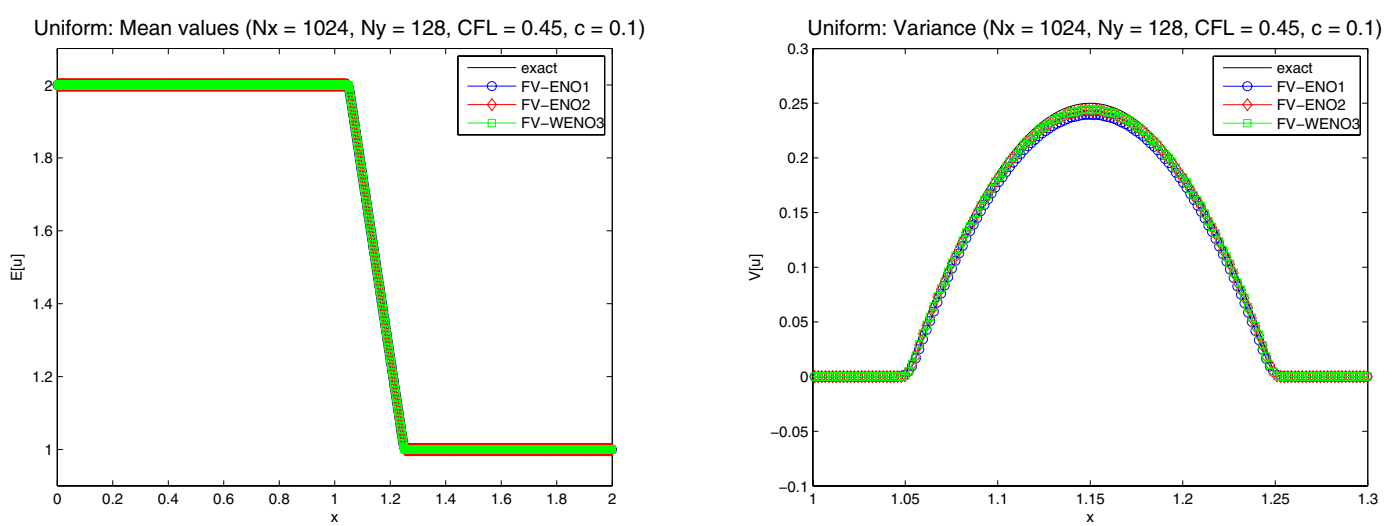

FigURE 1. Expectation and variance of the probabilistic shock profile, $y \sim \mathcal{U}[0.9 .1 .1]$.

The probability density function in terms of the random variable $y$ is easily verified to be

$$
\rho(y)=\left\{\begin{array}{l}
1 /(2 c), y \in\left[x_{0}-c, x_{0}+c\right] ; \\
0, \text { otherwise }
\end{array}\right.
$$

and the corresponding distribution function is

$$
P_{\mathcal{U}}(x)=\left\{\begin{array}{l}
0, x<x_{0}-c ; \\
\frac{1}{2 c}\left(x-x_{0}+c\right), x \in\left[x_{0}-c, x_{0}+c\right] ; \\
1, x>x_{0}+c .
\end{array}\right.
$$

The expectation of the random entropy solution $u$ is then

$$
\begin{aligned}
\mathbb{E}[u]=u_{L}+\left(u_{R}-u_{L}\right) P_{\mathcal{U}}(x-S t) & = \\
& =u_{L}+\left(u_{R}-u_{L}\right)\left\{\begin{array}{l}
0, x-S t<x_{0}-c ; \\
\frac{1}{2 c}\left(x-S t-x_{0}+c\right), x-S t \in\left[x_{0}-c, x_{0}+c\right] ; \\
1, x-S t>x_{0}+c .
\end{array}\right.
\end{aligned}
$$

Consider Figure 1 in which the mean and the variance of the probabilistic shock profiles computed by the Stochastic Finite Volume method [5] is plotted. In this figure, ENO-1 stands for the first order finite volume solver, ENO-2 and WENO-3 for the ENO/WENO solvers with piece-wise linear reconstruction). The values $N_{x}$ and $N_{y}$ refer to the number of cells in $x$ and $y$ coordinates, respectively.

Obviously, the function $\mathbb{E}[u]$ is continuous at points $x=S t+x_{0} \pm c$ for every $t>0$. However, pointwise derivatives at these points do not exist, meaning that $\mathbb{E}[u]$ is only in $C^{0}(\mathbb{R})$. It is easy to see that $\mathbb{E}[u] \in$ $C_{p w}^{\infty}\left(\mathbb{R}, \mathcal{P}_{t}\right)$ : the partition of $\mathbb{R}$ generated by the function $\rho$ is

$$
\mathcal{P}_{\rho}=\left\{\left(-\infty, x_{0}-c\right),\left(x_{0}-c, x_{0}+c\right),\left(x_{0}+c, \infty\right)\right\},
$$

and on each piece $I \in \mathcal{P}_{\rho}$ the density function $\rho \in C^{\infty}(\bar{I})$. The partition of $\mathbb{R}$ induced by the distribution function $P$ for every $t>0$ is

$$
\mathcal{P}_{t}=\left\{\left(-\infty, S t+x_{0}-c\right),\left(S t+x_{0}-c, x_{0}+c\right),\left(S t+x_{0}+c, \infty\right)\right\}
$$



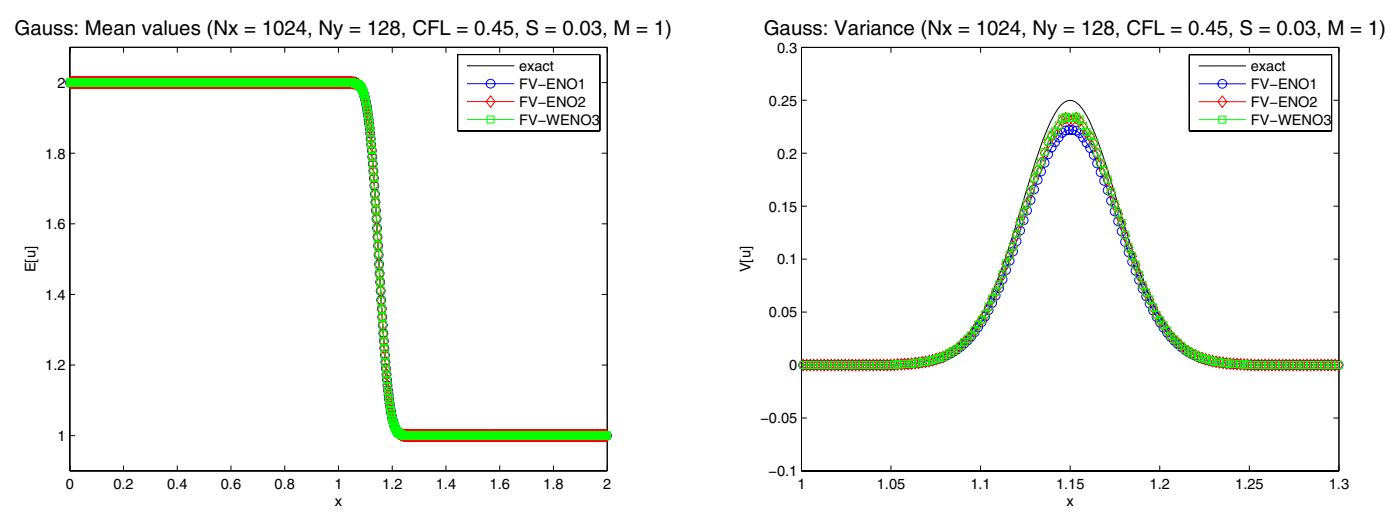

FiguRE 2. Expectation and variance of the probabilistic shock profile, $Y(\omega) \sim \mathcal{N}[1,0.03]$.

and $P \in C^{\infty}(\bar{I}) \forall I \in \mathcal{P}_{t}$. Consequently, by our definition of a piece-wise smooth function we have $\mathbb{E}[u] \in$ $C_{p w}^{\infty}\left(\mathbb{R}, \mathcal{P}_{t}\right)$.

\section{Example 3.12 (Normal distribution).}

Consider again the Riemann problem for the Burgers equation (3.8) with discontinuous initial data (3.9) assuming $Y(\omega) \sim \mathcal{N}[M, \sigma]$. The probability density function for the normal distribution is

$$
\rho(Y(\omega))=\frac{1}{\sqrt{2 \pi \sigma^{2}}} \mathrm{e}^{-\frac{(Y(\omega)-M)^{2}}{2 \sigma^{2}}} .
$$

Using the error function

$$
\operatorname{Erf}(x)=\frac{2}{\sqrt{\pi}} \int_{0}^{x} \mathrm{e}^{-y^{2}} \mathrm{~d} y,
$$

the distribution function for the normal distribution can be expressed as

$$
P_{\mathcal{N}}(x)=\frac{1}{2}\left[1+\operatorname{Erf}\left(\frac{x-M}{\sqrt{2 \sigma^{2}}}\right)\right],
$$

which is a smooth function in $\mathbb{R}$, that is $P_{\mathcal{N}} \in C^{\infty}(\mathbb{R})$ and therefore the expectation

$$
\mathbb{E}[u]=u_{L}+\left(u_{R}-u_{L}\right) P_{\mathcal{N}}(x-S t)=u_{L}+\frac{\left(u_{R}-u_{L}\right)}{2}\left[1+\operatorname{Erf}\left(\frac{x-M}{\sqrt{2 \sigma^{2}}}\right)\right]
$$

is also a smooth function, $\mathbb{E}[u] \in C^{\infty}(\mathbb{R})$.

The mean and the variance of the probabilistic shock profiles computed by the Stochastic Finite Volume method [5] is plotted in Figure 2.

\section{Example 3.13 (Atomic measure).}

Up to this point we have only considered the probabilistic spaces having the non-atomic measure $\mathbb{P}$ such that $\mathbb{P}(\mathrm{d} \omega)=\rho(y) \mathrm{d} y$ with $\rho \in\left(L^{\infty} \cap L^{1}\right)(\mathbb{R})$. However, it is worth considering the example of the atomic measure to show that the statistical moments of random entropy solutions to scalar conservation laws are not necessarily continuous in this case.

To this end, let the probability density function have the following form: $\rho(y)=\rho_{1}(y)+\frac{1}{2} \delta(y)$, where

$$
\rho_{1}(y)=\left\{\begin{array}{l}
1 / 4, y \in[-1,1] \\
0, \text { else }
\end{array}\right.
$$


Such $\rho(y)$ is atomic since it is not bounded at the point $y=0$ because of the presence of delta-function. As before, the mean value of the solution is given by (3.7) and its regularity is entirely determined by the distribution function $P(x-S t)$, which in our example takes the form

$$
P(x-S t)=\left\{\begin{array}{l}
0, x<S t-1 ; \\
\frac{1}{4}(x-S t+1), S t-1 \leqslant x<S t \\
\frac{1}{4}(x-S t+1)+\frac{1}{2} ; S t \leqslant x<S t+1 ; \\
1, x \geqslant S t+1 .
\end{array}\right.
$$

Apparently, the distribution function $P(x)$ is discontinuous at the point $x=0$ for any $t>0$, although the density function $\rho(y)$ could be considered as piece-wise differentiable, but not bounded at $y=0$. However, $\mathbb{E}[u] \in C_{p w}^{\infty}\left(\mathbb{R}, \mathcal{P}_{t}\right)$ with respect to the partition

$$
\mathcal{P}_{t}=\{(-\infty, S t-1),(S t-1, S t),(S t, S t+1),(S t+1, \infty)\} .
$$

\section{REgularity OF SPATIO-TEMPORAL CORRELATION FUNCTIONS}

To form the $n$-point correlation functions, form the tensor product of the pathwise random entropy solutions:

$$
\mathcal{C}^{n}[u]=u\left(x_{1}, t_{1}, \omega\right) \otimes u\left(x_{2}, t_{2}, \omega\right) \otimes \ldots \otimes u\left(x_{n}, t_{n}, \omega\right) .
$$

The $n$-point spacio-temporal correlation function is the expectation

$$
\begin{aligned}
\mathcal{M}^{n}[u] & =\mathbb{E}\left[\mathcal{C}^{n}[u]\right]=\mathbb{E}\left[u\left(x_{1}, t_{1}, \omega\right) \otimes \ldots \otimes u\left(x_{n}, t_{n}, \omega\right)\right] \\
& =\int_{\Omega} u\left(x_{1}, t_{1}, \omega\right) \otimes \ldots \otimes u\left(x_{n}, t_{n}, \omega\right) \mathbb{P}(\mathrm{d} \omega)=\int_{\mathbb{R}} u\left(x_{1}, t_{1}, y\right) \ldots u\left(x_{n}, t_{n}, y\right) \rho(y) \mathrm{d} y,
\end{aligned}
$$

where $y: \Omega \rightarrow \mathbb{R}$ is a random variable parametrizing the probability space $\Omega$.

Specifically, consider the scalar equation (3.1) with initial condition (3.4). The solution in this case is a shock wave of the form (3.5), which is equivalent (up to multiplication and addition of some constant) to the Heaviside function; therefore we can write

$$
u(x, t, y) \sim H(x-S t-y)=\bar{H}\left(y-y_{0}\right), y_{0}=x-S t .
$$

The $n$-point correlation function of $(4.2)$ is

$$
\mathcal{M}^{n}[u]=\int_{\mathbb{R}} u\left(x_{1}, t_{1}, y\right) \ldots u\left(x_{n}, t_{n}, y\right) \rho(y) \mathrm{d} y=\int_{\mathbb{R}} \bar{H}\left(y-y_{0}^{1}\right) \ldots \bar{H}\left(y-y_{0}^{n}\right) \rho(y) \mathrm{d} y
$$

where $y_{0}^{i}=x_{i}-S t_{i}, i=1, \ldots n$.

The integrand in (4.3) is non-zero only if all Heaviside functions $\bar{H}\left(y-y_{0}^{i}\right) \neq 0$, which is accomplished if $\tilde{y}=\min _{i}\left\{x_{i}-S t_{i}\right\}>y$. Hence, the expression for the $n$-point correlation function becomes

$$
\mathcal{M}^{n}[u]=\int_{\mathbb{R}} \bar{H}(y-\tilde{y}) \rho(y) \mathrm{d} y=P(\tilde{y})=P\left(\min _{i}\left\{x_{i}-S t_{i}\right\}\right) .
$$

This defines the partition of $\mathbb{R}$ induced by $\mathcal{M}^{n}[u]$ which we denote as $\mathcal{P}_{M}^{n}$. It will allow us to formulate the general theorem on the regularity of the correlation functions. The proof is based on the same principles as the proof of Theorems 3.1-3.10 for the expectation. 
Theorem 4.1. For every $k \in \mathbb{N}_{0}$ and $\rho \in C^{k}(\mathbb{R})$ and for every $t>0$ the $n$-point space-time correlation function $\mathcal{M}^{n}[u] \in C_{p w}^{k+1}\left(\mathbb{R}^{n}, \mathcal{P}_{M, t}^{n}\right)$. In particular, if $\rho \in C^{\infty}(\mathbb{R})$ then $\mathcal{M}^{n}[u] \in C_{p w}^{\infty}\left(\mathbb{R}^{n}, \mathcal{P}_{M, t}^{n}\right)$. Moreover, if $\rho \in C^{k, \alpha}(\mathbb{R})$ then $\mathcal{M}^{n}[u] \in C_{p w}^{k+1, \alpha}\left(\mathbb{R}^{n}, \mathcal{P}_{M, t}^{n}\right)$.

An obvious corollary from Theorem 4.1 is the following.

Corollary 4.2. The following statements hold:

1. Let $\rho \in C_{p w}^{k}\left(\mathbb{R}, \mathcal{P}_{\rho}\right)$. Then $\mathcal{M}^{n}[u] \in C_{p w}^{k+1}\left(\mathbb{R}^{n}, \mathcal{P}_{M, t}^{n}\right)$ for any $t>0$.

2. Let $\rho \in C_{p w}^{\infty}\left(\mathbb{R}, \mathcal{P}_{\rho}\right)$. Then $\mathcal{M}^{n}[u] \in C_{p w}^{\infty}\left(\mathbb{R}^{n}, \mathcal{P}_{M, t}^{n}\right)$ for any $t>0$.

3. Let $\rho \in C_{p w}^{k, \alpha}\left(\mathbb{R}, \mathcal{P}_{\rho}\right)$. Then $\mathcal{M}^{n}[u] \in C_{p w}^{k+1, \alpha}\left(\mathbb{R}^{n}, \mathcal{P}_{M, t}^{n}\right)$ for any $t>0$, where $\mathcal{P}_{M, t}^{n}=T_{S} \mathcal{P}_{M}^{n}$.

We illustrate the foregoing theorem by the same example.

\section{Example 4.3 (Two-point correlation function).}

Consider the scalar one-dimensional Burgers equation (3.8)-(3.9). We shall analyze in detail the regularity of the two-point space-time correlation function and prove that $\mathcal{M}^{2}[u] \in C_{p w}^{k+1}\left(\mathbb{R}^{2}, \mathcal{P}_{M}^{2}\right)$ for $\rho \in C^{k}\left(\mathbb{R}, \mathcal{P}_{\rho}\right)$ with given $t_{1}, t_{2}>0$, where

$$
\mathcal{M}^{2}[u]=\int_{-\infty}^{\infty} u\left(x_{1}, t_{1}, y\right) u\left(x_{2}, t_{2}, y\right) \rho(y) \mathrm{d} y .
$$

Given the solution of the form (3.5), its two-point correlation function is

$$
\begin{aligned}
\mathcal{M}^{2}[u] & =\int_{-\infty}^{\infty}\left(u_{L}+\left(u_{R}-u_{L}\right) H\left(x_{1}-S t_{1}-y\right)\right)\left(u_{L}+\left(u_{R}-u_{L}\right) H\left(x_{2}-S t_{2}-y\right)\right) \rho(y) \mathrm{d} y \\
& =u_{L}^{2}+u_{L}\left(u_{R}-u_{L}\right)\left(P\left(x_{1}-S t_{1}\right)+P\left(x_{2}-S t_{2}\right)\right)+\left(u_{R}-u_{L}\right)^{2} \tilde{P}\left(x_{1}-S t_{1}, x_{2}-S t_{2}\right),
\end{aligned}
$$

where

$$
\tilde{P}\left(x_{1}, x_{2}\right)=\int_{-\infty}^{\min \left\{x_{1}, x_{2}\right\}} \rho(y) \mathrm{d} y=P\left(\min \left\{x_{1}, x_{2}\right\}\right)=P\left(x_{1}\right)+\left(P\left(x_{2}\right)-P\left(x_{1}\right)\right) H\left(x_{1}-x_{2}\right) .
$$

Let $\rho \in C^{k}(\mathbb{R})$, then $P \in C^{k+1}(\mathbb{R})$. The regularity of the two-point correlation function in the whole $\mathbb{R}^{2}$ space is determined by the properties of the function $\tilde{P}\left(x_{1}-S t_{1}, x_{2}-S t_{2}\right)$ for given times $t_{1}$ and $t_{2}$. It is easy to check that the function $\tilde{P}\left(x_{1}-S t_{1}, x_{2}-S t_{2}\right)$ is continuous across the line $\Gamma: x_{1}-S t_{1}=x_{2}-S t_{2}$ on the $\left(x_{1}, x_{2}\right)$ plane, implying $\mathcal{M}^{2}[u] \in C^{0}\left(\mathbb{R}^{2}\right)$. The derivatives of $\mathcal{M}^{2}[u]$ are, however, discontinuous across the line $\Gamma$ : for instance,

$$
\begin{gathered}
\left.\frac{\partial}{\partial x_{1}} \mathcal{M}^{2}[u]\right|_{K_{1}}=u_{L}\left(u_{R}-u_{L}\right) \frac{\partial}{\partial x_{1}} P\left(x_{1}-S t_{1}\right)+\left(u_{R}-u_{L}\right)^{2} \frac{\partial}{\partial x_{1}} P\left(x_{1}-S t_{1}\right)=u_{R}\left(u_{R}-u_{L}\right) \rho\left(x_{1}-S t_{1}\right), \\
\left.\frac{\partial}{\partial x_{1}} \mathcal{M}^{2}[u]\right|_{K_{2}}=u_{L}\left(u_{R}-u_{L}\right) \frac{\partial}{\partial x_{1}} P\left(x_{1}-S t_{1}\right)+\left(u_{R}-u_{L}\right)^{2} \frac{\partial}{\partial x_{1}} P\left(x_{2}-S t_{2}\right)=u_{L}\left(u_{R}-u_{L}\right) \rho\left(x_{1}-S t_{1}\right) .
\end{gathered}
$$

Obviously, $\left.\frac{\partial}{\partial x_{1}} \mathcal{M}^{2}[u]\right|_{K_{1}} \neq\left.\frac{\partial}{\partial x_{1}} \mathcal{M}^{2}[u]\right|_{K_{2}}$ along $\Gamma$ unless $u_{L}=u_{R}$, which is a trivial case that we will not consider.

Moreover, it is possible to prove that $\mathcal{M}^{2}[u] \in C_{p w}^{k+1}\left(\mathbb{R}^{2}, \mathcal{P}_{M}^{2}\right)$ if $\rho \in C^{k}(\mathbb{R})$. Consider the following finite partition of $\mathbb{R}^{2}: \mathcal{P}_{M}^{2}=\left\{K_{j}\right\}_{j=1}^{2}$, where

$$
K_{1}=\left\{\left(x_{1}, x_{2}\right): x_{1}-x_{2}-S\left(t_{1}-t_{2}\right)<0\right\}, K_{2}=\left\{\left(x_{1}, x_{2}\right): x_{1}-x_{2}-S\left(t_{1}-t_{2}\right)>0\right\} .
$$


Obviously, $K_{1} \cap K_{2}=\emptyset, \mathbb{R}^{2}=\overline{K_{1} \cup K_{2}}$, and

$$
\tilde{P}\left(x_{1}-S t_{1}, x_{2}-S t_{2}\right)=\left\{\begin{array}{l}
P\left(x_{1}-S t_{1}\right) \text { on } K_{1} ; \\
P\left(x_{2}-S t_{2}\right) \text { on } K_{2} .
\end{array}\right.
$$

Therefore $\tilde{P}\left(x_{1}-S t_{1}, x_{2}-S t_{2}\right) \in C^{k+1}\left(K_{1}\right), \tilde{P}\left(x_{1}-S t_{1}, x_{2}-S t_{2}\right) \in C^{k+1}\left(K_{2}\right)$ and the same holds true for the two-point correlation function: $\mathcal{M}^{2}[u] \in C^{k+1}\left(K_{1}\right), \mathcal{M}^{2}[u] \in C^{k+1}\left(K_{2}\right)$. Then, taking into account Definition 3.7, $\mathcal{M}^{2}[u] \in C_{p w}^{k+1}\left(\mathbb{R}^{2}, \mathcal{P}_{M}^{2}\right) \cap C^{0}\left(\mathbb{R}^{2}\right)$.

Assume next in $(3.9) Y(\omega) \sim \mathcal{U}[0,1]$. Then $\mathcal{M}^{2}[u] \in C_{p w}^{\infty}\left(\mathbb{R}^{2}, \mathcal{P}_{M}^{2}\right) \cap C^{0}\left(\mathbb{R}^{2}\right)$. In this case we take $y=$ $x_{0}+c(2 Y(\omega)-1)$ so that $y \sim \mathcal{U}\left[x_{0}-c, x_{0}+c\right]$. Then the two-point correlation function can be expressed as

$$
\mathcal{M}^{2}[u]=u_{L}^{2}+u_{L}\left(u_{R}-u_{L}\right)\left(I_{1}\left(x_{1}\right)+I_{2}\left(x_{2}\right)\right)+\left(u_{R}-u_{L}\right)^{2} I_{3}\left(x_{1}, x_{2}\right),
$$

where we set $x_{0}^{*}=\min \left\{x_{1}-S t_{1}, x_{2}-S t_{2}\right\}$ and

$$
\begin{aligned}
& I_{1}\left(x_{1}\right)=\int_{0}^{1} H\left(x_{1}-S t_{1}-x_{0}(y)\right) \mathrm{d} y=\frac{1}{2 c} \int_{-c}^{c} H\left(x_{1}-S t_{1}-x_{0}\right) \mathrm{d} x_{0} \\
& =\left\{\begin{array}{l}
0, x_{1} \leqslant S t_{1}-c ; \\
\frac{1}{2 c}\left(x_{1}-S t_{1}+c\right), x_{1} \in\left(S t_{1}-c, S t_{1}+c\right) ; \\
1, x_{1} \geqslant S t_{1}+c,
\end{array}\right. \\
& I_{2}\left(x_{2}\right)=\int_{0}^{1} H\left(x_{2}-S t_{2}-x_{0}(y)\right) \mathrm{d} y=\left\{\begin{array}{l}
0, x_{2} \leqslant S t_{2}-c ; \\
\frac{1}{2 c}\left(x_{2}-S t_{2}+c\right), x_{2} \in\left(S t_{2}-c, S t_{2}+c\right) ; \\
1, x_{2} \geqslant S t_{2}+c,
\end{array}\right. \\
& I_{3}\left(x_{1}, x_{2}\right)=\int_{0}^{1} H\left(x_{1}-S t_{1}-x_{0}(y)\right) H\left(x_{2}-S t_{2}-x_{0}(y)\right) \mathrm{d} y=\left\{\begin{array}{l}
0, x_{0}^{*} \leqslant-c ; \\
\frac{1}{2 c}\left(x_{0}^{*}+c\right), x_{0}^{*} \in(-c, c) ; \\
1, x_{0}^{*} \geqslant c,
\end{array}\right.
\end{aligned}
$$

To analyze the regularity of $\mathcal{M}^{2}[u]$ we consider the partition of $\mathbb{R}^{2}$ induced by $\mathcal{M}^{2}[u]$.

Assume first that $x_{0}^{*}=x_{1}-S t_{1}<x_{2}-S t_{2}$. We have the following subdomains for the integral $I_{1}\left(x_{1}\right)$, depending on the position of $x_{0}^{*}$ defining $I_{3}\left(x_{1}, x_{2}\right)$ also uniquely.

1. $x_{1}-S t_{1} \leqslant-c \Rightarrow I_{1}\left(x_{1}\right)=0, I_{3}\left(x_{1}, x_{2}\right)=0$.

For $I_{2}\left(x_{2}\right)$ there are three subdomains:

(a) $x_{2}-S t_{2} \leqslant-c \Rightarrow I_{2}\left(x_{2}\right)=0 \Rightarrow \mathcal{M}_{1}^{2}[u]=u_{L}^{2}$.

(b) $\left|x_{2}-S t_{2}\right|<c \Rightarrow I_{2}\left(x_{2}\right)=\frac{1}{2 c}\left(x_{2}-S t_{2}+c\right) \Rightarrow \mathcal{M}_{2}^{2}[u]=u_{L}^{2}+\frac{1}{2 c} u_{L}\left(u_{R}-u_{L}\right)\left(x_{2}-S t_{2}+c\right)$.

(c) $x_{2}-S t_{2} \geqslant c \Rightarrow I_{2}\left(x_{2}\right)=1 \Rightarrow \mathcal{M}_{3}^{2}[u]=u_{L} u_{R}$.

2. $\left|x_{1}-S t_{1}\right|<c \Rightarrow I_{1}\left(x_{1}\right)=\frac{1}{2 c}\left(x_{1}-S t_{1}+c\right), I_{3}\left(x_{1}, x_{2}\right)=\frac{1}{2 c}\left(x_{1}-S t_{1}+c\right)$.

In this case there are two possible subdomains for $I_{2}\left(x_{2}\right)$ since $x_{2}-S t_{2}>x_{1}-S t_{1}>-c$.

(a) $\left|x_{2}-S t_{2}\right|<c \Rightarrow I_{2}\left(x_{2}\right)=\frac{1}{2 c}\left(x_{2}-S t_{2}+c\right) \Rightarrow$

$$
\mathcal{M}_{4}^{2}=u_{L}^{2}+\frac{1}{2 c} u_{L}\left(u_{R}-u_{L}\right)\left(x_{1}-S t_{1}+x_{2}-S t_{2}\right)+\frac{1}{2 c}\left(u_{R}-u_{L}\right)^{2}\left(x_{1}-S t_{1}+c\right) .
$$

(b) $x_{2}-S t_{2} \geqslant c \Rightarrow I_{2}\left(x_{2}\right)=1 \Rightarrow$

$$
\mathcal{M}_{5}^{2}=u_{L}^{2}+u_{L}\left(u_{R}-u_{L}\right)\left(\frac{1}{2 c}\left(x_{1}-S t_{1}+c\right)+1\right)+\frac{1}{2 c}\left(u_{R}-u_{L}\right)^{2}\left(x_{1}-S t_{1}+c\right) .
$$




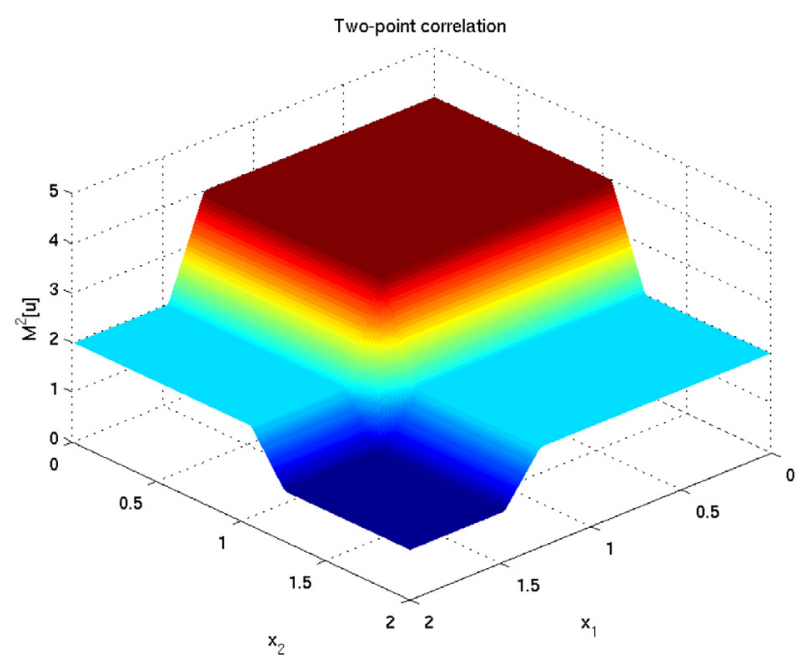

Figure 3. Two-point correlation function for the Burgers equation with uncertain initial data.

3. $x_{1}-S t_{1} \geqslant c \Rightarrow I_{1}\left(x_{1}\right)=1, I_{3}\left(x_{1}, x_{2}\right)=1, I_{2}\left(x_{2}\right)=1 \Rightarrow \mathcal{M}_{6}^{2}[u]=u_{R}^{2}$.

Now assume that $x_{0}^{*}=x_{2}-S t_{2}<x_{1}-S t_{1}$. We have the following subdomains for $I_{2}\left(x_{2}\right)$.

1. $x_{2}-S t_{2} \leqslant-c \Rightarrow I_{2}\left(x_{2}\right)=0, I_{3}\left(x_{1}, x_{2}\right)=0$.

For $I_{1}\left(x_{1}\right)$ there are three subdomains:

(a) $x_{1}-S t_{1} \leqslant-c \Rightarrow I_{1}\left(x_{1}\right)=0 \Rightarrow \mathcal{M}_{7}^{2}[u]=u_{L}^{2}$.

(b) $\left|x_{1}-S t_{1}\right|<c \Rightarrow I_{1}\left(x_{1}\right)=\frac{1}{2 c}\left(x_{1}-S t_{1}+c\right) \Rightarrow \mathcal{M}_{8}^{2}[u]=u_{L}^{2}+\frac{1}{2 c} u_{L}\left(u_{R}-u_{L}\right)\left(x_{1}-S t_{1}+c\right)$.

(c) $x_{1}-S t_{1} \geqslant c \Rightarrow I_{1}\left(x_{1}\right)=1 \Rightarrow \mathcal{M}_{9}^{2}[u]=u_{L} u_{R}$.

2. $\left|x_{2}-S t_{2}\right|<c \Rightarrow I_{2}\left(x_{2}\right)=\frac{1}{2 c}\left(x_{2}-S t_{2}+c\right), I_{3}\left(x_{1}, x_{2}\right)=\frac{1}{2 c}\left(x_{2}-S t_{2}+c\right)$.

There are two possible subdomains for $I_{1}\left(x_{1}\right)$ since $x_{1}-S t_{1}>x_{2}-S t_{2}>-c$.

(a) $\left|x_{1}-S t_{1}\right|<c \Rightarrow I_{1}\left(x_{1}\right)=\frac{1}{2 c}\left(x_{1}-S t_{1}+c\right) \Rightarrow$

$$
\mathcal{M}_{10}^{2}=u_{L}^{2}+\frac{1}{2 c} u_{L}\left(u_{R}-u_{L}\right)\left(x_{1}-S t_{1}+x_{2}-S t_{2}\right)+\frac{1}{2 c}\left(u_{R}-u_{L}\right)^{2}\left(x_{2}-S t_{2}+c\right) .
$$

(b) $x_{1}-S t_{1} \geqslant c \Rightarrow I_{1}\left(x_{1}\right)=1 \Rightarrow$

$$
\mathcal{M}_{11}^{2}=u_{L}^{2}+u_{L}\left(u_{R}-u_{L}\right)\left(\frac{1}{2 c}\left(x_{2}-S t_{2}+c\right)+1\right)+\frac{1}{2 c}\left(u_{R}-u_{L}\right)^{2}\left(x_{2}-S t_{2}+c\right) .
$$

3. $x_{2}-S t_{2} \geqslant c \Rightarrow I_{2}\left(x_{2}\right)=1, I_{3}\left(x_{1}, x_{2}\right)=1, I_{1}\left(x_{1}\right)=1 \Rightarrow \mathcal{M}_{12}^{2}[u]=u_{R}^{2}$.

In summary, we have constructed the partition $\mathcal{P}_{M}^{2}$ of $\mathbb{R}^{2}$ such that $\mathcal{M}^{2}[u] \in C_{p w}^{\infty}\left(\mathbb{R}^{2}, \mathcal{P}_{M}^{2}\right)$.

Figure 3 illustrates the two-point correlation function for the Burgers equation (3.8) with uncertain initial data (3.10) and $y \sim \mathcal{U}[0.9,1.1]$. To approximate the two-point correlation function we have computed the pathwise solutions $u\left(x, t_{1}, y\right)$ and $u\left(x, t_{2}, y\right)$ at $t_{1}=0.1$ and $t_{2}=0.25$ using the high-order SFV method based on the piece-wise quadratic 5th order WENO reconstruction on the mesh of 256 cells in $x$ and 64 cells in $y$. Then we have approximated the correlation function according to (4.5) by the mid-point quadrature rule in each mesh cell.

Obviously, the correlation function is piece-wise smooth and continuous in $\mathbb{R}^{2}$. 


\section{Regularity OF POLYNOMial MOMENTS}

In this section we follow $[9,14]$ in the definition of the generalized polynomial chaos expansion (gPC) and the formulation of the assumptions under which the gPC expansion converges to a desired random variable, see also $[10,11,18]$. We then address the question of the regularity of the expansion coefficients, also called polynomial moments.

Consider the probability space $(\Omega, \mathcal{F}, \mathbb{P})$ defined in Section 2.1 , with probability measure $\mathbb{P}$. Consider the chaos expansion with respect to a countable sequence $\left\{\xi_{m}\right\}_{m \in \mathbb{N}}$ of basic random variables satisfying the following assumptions:

\section{Assumption 5.1.}

(1) Each basic random variable $\xi_{m}$ possesses finite moments of all orders, i.e. $\mathbb{E}\left[\left|\xi_{m}\right|^{k}\right]<\infty$ for all $k, m \in \mathbb{N}$.

(2) The distribution functions $P_{\xi_{m}}(x)=\mathbb{P}\left(\xi_{m}<x\right)$ of the basic random variables are continuous.

We next restrict ourselves to the expansions in a single basic random variable $\xi$ with distribution function $P_{\xi}$ which satisfies Assumption 5.1. For any random variable $\eta \in L^{2}(\Omega, \mathcal{F}, \mathbb{P})$ which is measurable with respect to $\xi$ there exists a measurable function $f: \mathbb{R} \rightarrow \mathbb{R}$ such that $\eta=f(\xi)$. To expand $\eta$ in the gPC series one needs to construct the set of orthonormal polynomials $\left\{p_{n}\right\}_{n \in \mathbb{N}_{0}}$ associated with the distribution function $P_{\xi}$, which is possible since all the moments of $\xi$ are finite by Assumption 5.1.

Definition 5.2. The moment problem is uniquely solvable for a probability distribution on $(\mathbb{R}, \mathcal{B}(\mathbb{R})$ ) (or the distribution is determinate), if the distribution function is uniquely defined by the sequence of its moments

$$
\mu_{k}=\mathbb{E}\left[\xi^{k}\right]=\int_{\mathbb{R}} x^{k} P_{\xi}(\mathrm{d} x), \quad k \in \mathbb{N}_{0} .
$$

The following theorem justifies the existence of the gPC expansion [9].

Theorem 5.3. The sequence of orthogonal polynomials associated with a real random variable $\xi$ satisfying Assumption 5.1 is dense in $L^{2}\left(\mathbb{R}, \mathcal{B}(\mathbb{R}), P_{\xi}\right)$ if and only if the moment problem is uniquely solvable for its distribution.

Thus, if the conditions of Theorem 5.3 are satisfied, the sequence of random variables $\left\{p_{n}(\xi)\right\}_{n \in \mathbb{N}_{0}}$ constitutes an orthonormal basis of the Hilbert space $L^{2}(\Omega, \mathcal{F}, \mathbb{P})$, and each element of this space can be expanded with respect to this basis:

$$
\eta=f(\xi)=\sum_{k=0}^{\infty} a_{k} p_{k}(\xi)
$$

and the coefficients $a_{k}$ can be calculated as

$$
a_{k}=\mathbb{E}\left[\eta p_{k}(\xi)\right]=\int_{\mathbb{R}} f(x) p_{n}(x) P_{\xi}(\mathrm{d} x), \quad k \in \mathbb{N}_{0} .
$$

Consider now the solution to the one-dimensional scalar conservation law (2.10) with random initial data (2.11). Assuming the existence of the random variable such that $y=Y(\omega) \in \mathbb{R}$ satisfying the Assumption 5.1 with continuous distribution function $P(x)=\mathbb{P}(\{y<x\})$ such that there exists a probability density function $\rho(y)=d \mathbb{P}(\omega) / \mathrm{d} y$, we can write the gPC expansion (5.1) in $y$ for the stochastic function $\left.u(x, t, y)\right|_{y=Y(\omega)}$ :

$$
u(x, t, y)=\sum_{\alpha=0}^{\infty} u_{\alpha}(x, t) \varphi_{\alpha}(y)
$$


where $\left\{\varphi_{\alpha}\right\}_{\alpha \in \mathbb{N}_{0}}$ is the set of orthonormal polynomials associated with the distribution function $P$ :

$$
\left\langle\varphi_{\alpha}, \varphi_{\beta}\right\rangle=\int_{\mathbb{R}} \varphi_{\alpha}(y) \varphi_{\beta}(y) \rho(y) \mathrm{d} y=\delta_{\alpha \beta} .
$$

The coefficients of the gPC expansion are deterministic functions of $x$ and $t$ and are defined as follows:

$$
u_{\alpha}(x, t)=\mathbb{E}\left[\varphi_{\alpha} u\right]=\int_{\mathbb{R}} u(x, t, y) \varphi_{\alpha}(y) \rho(y) \mathrm{d} y .
$$

Consider next the one-dimensional scalar conservation law (3.1) and initial data (3.4) with $y=x_{0}+Y(\omega)$. The solution is equivalent to (4.2) up to some constant which does not affect the regularity properties. To study the regularity of the expansion coefficients $u_{\alpha}(x, t)$, first rewrite (5.4) for the one-dimensional shock solution and apply integration by parts:

$$
\begin{aligned}
u_{\alpha}(x, t) & =\int_{-\infty}^{\infty} \bar{H}\left(y-y_{0}\right) \varphi_{\alpha}(y) \rho(y) \mathrm{d} y=\int_{-\infty}^{\infty} \bar{H}\left(y-y_{0}\right) \varphi_{\alpha}(y) P^{\prime}(y) \mathrm{d} y \\
& =\left.\int_{-\infty}^{\infty} \bar{H}_{\varphi, \alpha}\left(y-y_{0}\right) P^{\prime}(y) \mathrm{d} y \bar{H}_{\varphi, \alpha}\left(y-y_{0}\right) P(y)\right|_{-\infty} ^{\infty}-\int_{-\infty}^{\infty} \bar{H}_{\varphi, \alpha}^{\prime}\left(y-y_{0}\right) P(y) \mathrm{d} y \\
& =-\int_{-\infty}^{\infty}\left\{\bar{H}^{\prime}\left(y-y_{0}\right) \varphi_{\alpha}(y)+\bar{H}\left(y-y_{0}\right) \varphi_{\alpha}^{\prime}(y)\right\} P(y) \mathrm{d} y \\
& =\int_{-\infty}^{\infty} \delta\left(y-y_{0}\right) \varphi_{\alpha}(y) P(y) \mathrm{d} y-\int_{-\infty}^{\infty} \bar{H}\left(y-y_{0}\right) \varphi_{\alpha}^{\prime}(y) P(y) \mathrm{d} y \\
& =P(x-S t) \varphi_{\alpha}(x-S t)-\int_{-\infty}^{x t} P(y) \varphi_{\alpha}^{\prime}(y) \mathrm{d} y
\end{aligned}
$$

where we have denoted $\bar{H}_{\varphi, \alpha}=\bar{H}\left(y-y_{0}\right) \varphi_{\alpha}(y)$.

Note that the functions $\varphi_{\alpha}(y)$ are polynomials and hence they belong to $C^{\infty}(\mathbb{R})$. The second, integral term in (5.5) apparently is at least as smooth as $P(y)$. Therefore, these terms do not affect the smoothness of the coefficient $u_{\alpha}(x, t)$, which is entirely defined by the regularity of the distribution function $P$. Thus, we can summarize the results which are analogous to the results for the expectation and statistical moments.

Theorem 5.4. For any $k \in \mathbb{N}_{0}$ and any $t>0$, if $\rho \in C^{k}(\mathbb{R})$ then $u_{\alpha} \in C^{k+1}(\mathbb{R}), \alpha \geqslant 0$. In particular, if $\rho \in C^{\infty}(\mathbb{R})$ then $u_{\alpha} \in C^{\infty}(\mathbb{R}), \alpha \geqslant 0$. For $\rho \in L^{\infty}(\mathbb{R})$ the polynomial moment $u_{\alpha} \in W^{1, \infty}(\mathbb{R}), \alpha \geqslant 0$.

Corollary 5.5. The following statements are valid:

1. If $\rho \in C_{p w}^{k}\left(\mathbb{R}, \mathcal{P}_{\rho}\right)$, then $u_{\alpha} \in C_{p w}^{k+1}\left(\mathbb{R}, \mathcal{P}_{t}\right), \alpha \geqslant 0$, for any $t>0$.

2. If $\rho \in C_{p w}^{\infty}\left(\mathbb{R}, \mathcal{P}_{\rho}\right)$, then $u_{\alpha} \in C_{p w}^{\infty}\left(\mathbb{R}, \mathcal{P}_{t}\right), \alpha \geqslant 0$, for any $t>0$.

3. If $\rho \in C_{p w}^{k, \gamma}\left(\mathbb{R}, \mathcal{P}_{\rho}\right)$, then $u_{\alpha} \in C_{p w}^{k+1, \gamma}\left(\mathbb{R}, \mathcal{P}_{t}\right), \alpha \geqslant 0$, for any $t>0$. 


\section{Generalizations}

\subsection{Linear hyperbolic systems}

The results obtained in preceding sections for one-dimensional scalar conservation law can be easily extrapolated onto linear systems. Consider the linear hyperbolic system

$$
\frac{\partial \mathbf{U}}{\partial t}+\mathbf{A} \frac{\partial \mathbf{U}}{\partial x}=\mathbf{0}, x \in \mathbb{R}, t>0
$$

with Riemann initial data and uncertain initial discontinuity position given in the parametrized form

$$
\mathbf{U}(x, 0, y)=\mathbf{U}_{0}(x, y)=\left\{\begin{array}{l}
\mathbf{U}_{L}, x<y \\
\mathbf{U}_{R}, x>y
\end{array}\right.
$$

where $\mathbf{U}=\left[u_{1}, \ldots, u_{m}\right]^{T}$ is the solution vector and $\mathbf{A}$ is the constant coefficient matrix. It is possible to diagonalize the system (6.1) by transforming it to new variables (Riemann invariants) $\mathbf{W}=\mathbf{R}^{-1} \mathbf{U}$, where $\mathbf{R}=$ $\left[\mathbf{r}_{1}, \ldots, \mathbf{r}_{m}\right]$ is the matrix of right eigenvectors of $\mathbf{A}$ such that $\mathbf{A}=\mathbf{R} \boldsymbol{\Lambda} \mathbf{R}^{-1}$ with the matrix $\boldsymbol{\Lambda}=\operatorname{diag}\left(\lambda_{1}, \ldots, \lambda_{m}\right)$ being the diagonal matrix of eigenvalues of $\mathbf{A}$.

Hence, in Riemann invariants the system and the initial condition can be recast as

$$
\begin{gathered}
\frac{\partial \mathbf{W}}{\partial t}+\Lambda \frac{\partial \mathbf{W}}{\partial x}=\mathbf{0}, \\
\mathbf{W}(x, 0, y)=\mathbf{W}_{0}(x, y)=\left\{\begin{array}{l}
\mathbf{W}_{L}=\mathbf{R}^{-1} \mathbf{U}_{L}, x<y ; \\
\mathbf{W}_{R}=\mathbf{R}^{-1} \mathbf{U}_{R}, x>y
\end{array}\right.
\end{gathered}
$$

or, in component-wise form for $i=1, \ldots, m$

$$
\begin{gathered}
\frac{\partial w_{i}}{\partial t}+\lambda_{i} \frac{\partial w_{i}}{\partial x}=0, \\
w_{i}(x, 0, y)=w_{i}^{0}(x, y)= \begin{cases}w_{L}^{i}, & x<y ; \\
w_{R}^{i}, & x>y .\end{cases}
\end{gathered}
$$

Obviously, for each $i$ the equations (6.5)-(6.6) form the linear transport problem, whose exact solution is a shock wave moving with speed $\lambda_{i}$,

$$
w_{i}(x, t, y)=w_{L}^{i}+\left(w_{R}^{i}-w_{L}^{i}\right) H\left(x-\lambda_{i} t-y\right) .
$$

The solution in initial variables is then a linear combination of shocks of the form (6.7):

$$
\mathbf{U}(x, t, y)=\mathbf{R W}=\sum_{i=1}^{m} w_{i}(x, t, y) \mathbf{r}_{i}=\sum_{i=1}^{m}\left(w_{L}^{i}+\left(w_{R}^{i}-w_{L}^{i}\right) H\left(x-\lambda_{i} t-y\right)\right) \mathbf{r}_{i} .
$$

Therefore, all the regularity statements obtained for scalar equations are also valid for linear systems.

Remark 6.1. Similar results are also anticipated in the case of quasi-linear one-dimensional systems of conservation laws. 

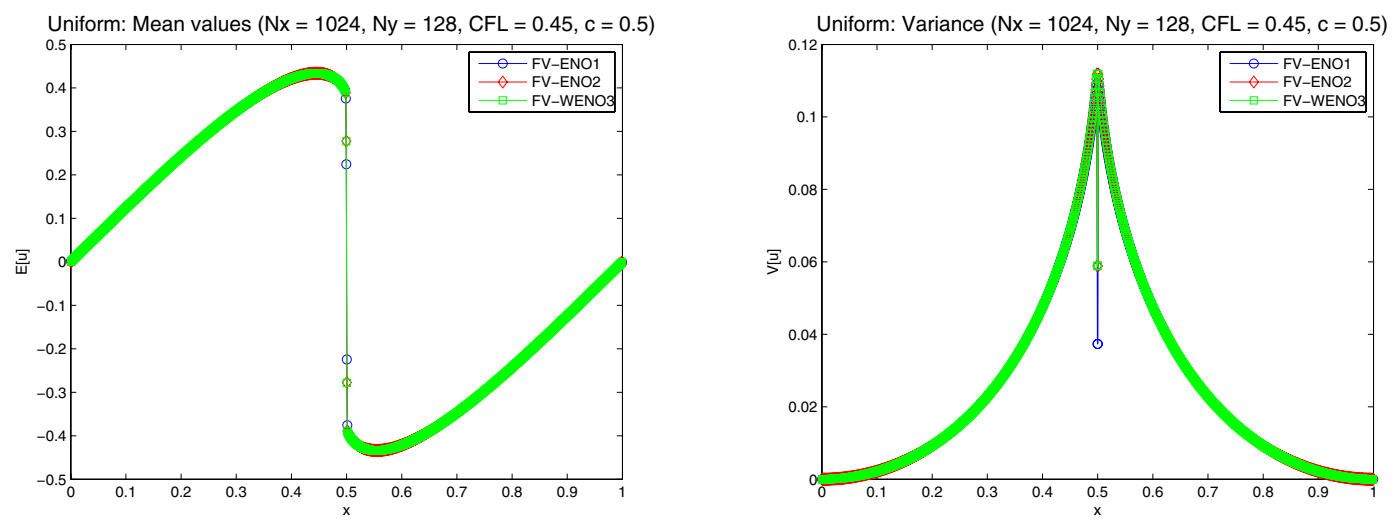

Figure 4. Expectation and variance of the random shock solution, $Y(\omega) \sim \mathcal{U}[0,1]$.

\subsection{Uncertain shock amplitude}

In this section we demonstrate the intrinsic difference between the probabilistic shock profile generated by the Riemann initial data with uncertain shock position having smooth statistics and the probabilistic shock profile with shock statistics. Consider the scalar one-dimensional Burgers equation

$$
\frac{\partial u}{\partial t}+u \frac{\partial u}{\partial x}=0
$$

with the initial condition having an uncertain amplitude:

$$
u_{0}(x, \omega)=Y(\omega) \sin (2 \pi x),
$$

where $Y(\omega)$ will be distributed either uniformly or normally,

$$
Y(\omega) \sim \mathcal{U}[-c, c] \text { or } Y(\omega) \sim \mathcal{N}[M, \sigma] .
$$

For such kind of problems the previously derived regularity theorems are no longer valid: the stationary shock is generated at certain time moment at $x=0.5$, and this shock remains present in the physical space. What is more, the existence of the shock does not depend on the regularity of the probability density function, which is illustrated in Figures 4-5. The numerical results were obtained by the Stochastic Finite Volume method combined with various deterministic solvers. As before, ENO-1 stands for the first order finite volume solver, ENO-2 and WENO-3 for the ENO/WENO solvers with piece-wise linear reconstruction). The values $N_{x}$ and $N_{y}$ refer to the number of cells in $x$ and $y$ coordinates, respectively.

\section{Convergence Rates of high order Stochastic Galerkin-FVM schemes}

Here, we explore the implications of the increased regularity of statistical moments of random entropy solutions on possible high order schemes for their numerical approximations. Clearly, schemes based on path-wise approximation and sample averaging as proposed, for example, in [3,24], will have to numerically resolve the discontinuities in the path-wise realizations of random entropy solutions. Accordingly, their potential high order convergence is offset by the lack of regularity of pathwise realizations of the random entropy solutions. To exploit the regularity of statistical moments requires, therefore, their direct approximation by FVM. To this end, new deterministic systems of hyperbolic conservation laws satisfied by these moments need to be derived analytically prior to discretization. This is possible, for example, by the so-called Stochastic Galerkin methods recently 

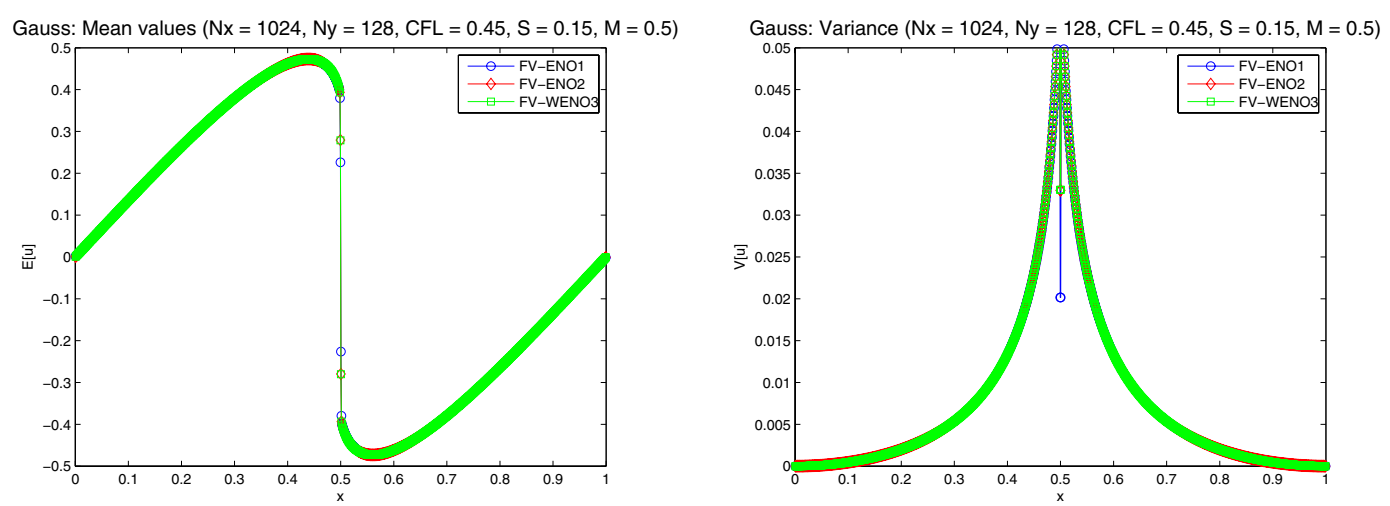

FIGURE 5. Expectation and variance of the random shock solution, $Y(\omega) \sim \mathcal{N}[0.5,0.15]$.

proposed in $[6,7,19-23]$. We briefly recapitulate the development here, and report numerical studies exhibiting maximal order of convergence for a number of numerical schemes for the resulting hyperbolic systems.

The results of Theorem 5.4 can be generalized to the linear systems case

$$
\begin{aligned}
& \frac{\partial \mathbf{U}}{\partial t}+\frac{\partial \mathbf{F}(\mathbf{U})}{\partial x}=\mathbf{0}, \mathbf{F}=\mathbf{A} \mathbf{U}, x \in(a, b), t>0 ; \\
& \mathbf{U}(x, 0, y)=\mathbf{U}_{0}(x, y), x \in(a, b), y \in Y \subset \mathbb{R},
\end{aligned}
$$

with uncertain initial shock location $\mathbf{U}_{0}(x, y)=H(x-y)$, where the shock position is distributed with the probability density $\rho(y)$.

Theorem 7.1. For a given probability density function $\rho$, any $k \in \mathbb{N}_{0}$ and any $t>0$ holds:

1. If $\rho \in C^{k}(\mathbb{R})$ then $\mathbf{U}_{\alpha} \in C^{k+1}(\mathbb{R}), \alpha \geqslant 0$. In particular, if $\rho \in C^{\infty}(\mathbb{R})$ then $\mathbf{U}_{\alpha} \in C^{\infty}(\mathbb{R}), \alpha \geqslant 0$. For $\rho \in L^{\infty}(\mathbb{R})$ the coefficient $\mathbf{U}_{\alpha} \in W^{1, \infty}(\mathbb{R}), \alpha \geqslant 0$.

2. If $\rho \in C_{p w}^{k}\left(\mathbb{R}, \mathcal{P}_{\rho}\right)$, then $\mathbf{U}_{\alpha} \in C_{p w}^{k+1}\left(\mathbb{R}, \mathcal{P}_{t}\right), \alpha \geqslant 0$, for any $t>0$.

3. If $\rho \in C_{p w}^{\infty}\left(\mathbb{R}, \mathcal{P}_{\rho}\right)$, then $\mathbf{U}_{\alpha} \in C_{p w}^{\infty}\left(\mathbb{R}, \mathcal{P}_{t}\right), \alpha \geqslant 0$, for any $t>0$.

4. If $\rho \in C_{p w}^{k, \gamma}\left(\mathbb{R}, \mathcal{P}_{\rho}\right)$, then $\mathbf{U}_{\alpha} \in C_{p w}^{k+1, \gamma}\left(\mathbb{R}, \mathcal{P}_{t}\right), \alpha \geqslant 0$, for any $t>0$.

Proof. The solution of the linear hyperbolic system with Riemann initial data is a linear combination of the invariants:

$$
\mathbf{U}(x, t, y)=\sum_{i=1}^{m} w_{i}(x, t, y) \mathbf{r}_{i},
$$

where each invariant $w_{i}(x, t, y)$ is a solution of the linear advection equation

$$
\frac{\partial w_{i}}{\partial t}+\lambda_{i} \frac{\partial w_{i}}{\partial x}=0
$$

The gPC expansion for $w_{i}(x, t, y)$ has the form

$$
w_{i}(x, t, y)=\sum_{\alpha=0}^{\infty} w_{i, \alpha}(x, t) \varphi_{\alpha}(y)
$$

where we choose the system of basis gPC polynomials $\left\{\varphi_{\alpha}\right\}, \alpha \in \mathbb{N}_{0}$ to be orthonormal with respect to the probability measure:

$$
\int_{Y} \varphi_{\alpha}(y) \varphi_{\beta}(y) \rho(y) \mathrm{d} y=\delta_{\alpha \beta},
$$

such that the equations for the gPC coefficients become fully decoupled. 
Then the gPC expansion for the solution of the linear system (7.1)-(7.2) is

$$
\mathbf{U}(x, t, y)=\sum_{\alpha=0}^{\infty} \mathbf{U}_{\alpha}(x, t) \varphi_{\alpha}(y)=\sum_{i=1}^{m} \sum_{\alpha=0}^{\infty} w_{i, \alpha}(x, t) \varphi_{\alpha}(y) \mathbf{r}_{i}=\sum_{\alpha=0}^{\infty}\left(\sum_{i=1}^{m} w_{i, \alpha}(x, t) \mathbf{r}_{i}\right) \varphi_{\alpha}(y),
$$

which is a linear combination of the gPC coefficients for the invariants, for which Theorem 5.4 can be applied directly.

Therefore, our smoothness results are valid for the solution of the linear hyperbolic systems.

\subsection{Stochastic Galerkin discretization}

In this section, we describe the construction of the Stochastic Galerkin Finite Volume method (sGFVM) for systems of hyperbolic conservation laws in one space dimension, i.e. for

$$
\begin{aligned}
& \frac{\partial \mathbf{U}}{\partial t}+\frac{\partial \mathbf{F}(\mathbf{U})}{\partial x}=\mathbf{0}, x \in(a, b), t>0 ; \\
& \mathbf{U}(x, 0, y)=\mathbf{U}_{0}(x, y), x \in(a, b), y \in Y \subset \mathbb{R} .
\end{aligned}
$$

The sGFVM aims to approximate the coefficients of the truncated gPC expansion

$$
u(x, t, y)=\sum_{k=0}^{N} \mathbf{U}_{k}(x, t) \varphi_{k}(y)
$$

where $\left\{\varphi_{k}\right\}, k=0, \ldots, N$ is a system of basis polymonials on $Y$. Note that these polynomials are not necessarily orthogonal with respect to the probability density function $\rho(y)$.

Multiplying the equations (7.3)-(7.4) by the basis function $\varphi_{i}(y)$ and integrating the result over $Y$ we obtain

$$
\begin{aligned}
& \int_{Y} \frac{\partial \mathbf{U}}{\partial t} \varphi_{i}(y) \rho(y) \mathrm{d} y+\int_{Y} \frac{\partial \mathbf{F}(\mathbf{U})}{\partial x} \varphi_{i}(y) \rho(y) \mathrm{d} y=\mathbf{0}, \\
& \int_{Y} \mathbf{U}(x, 0, y) \varphi_{i}(y) \rho(y) \mathrm{d} y=\int_{Y} \mathbf{U}_{0}(x, y) \varphi_{i}(y) \rho(y) \mathrm{d} y,
\end{aligned}
$$

which after simple algebraic manipulations reduces to

$$
\begin{aligned}
& \sum_{k=0}^{N} \frac{\partial \mathbf{U}_{k}}{\partial t} M_{k i}+\frac{\partial}{\partial x} \int_{Y} \mathbf{F}(\mathbf{U}) \varphi_{i}(y) \rho(y) \mathrm{d} y=\mathbf{0}, \\
& \sum_{k=0}^{N} \mathbf{U}_{k}^{(0)} M_{k i}=\int_{Y} \mathbf{U}_{0}(x, y) \varphi_{i}(y) \rho(y) \mathrm{d} y .
\end{aligned}
$$

where $\mathbf{U}_{k}^{(0)}=\mathbf{U}_{k}(x, 0)$ and $M_{k i}$ are the mass matrix components,

$$
M_{k i}=\int_{Y} \varphi_{k}(y) \varphi_{i}(y) \rho(y) \mathrm{d} y
$$

The resulting form of the Galerkin system (7.8)-(7.9) depends on the concrete form of the flux function $\mathbf{F}(\mathbf{U})$. 


\subsection{Convergence analysis for linearized Euler equations}

In this section, we apply the sGFVM for the numerical solution of the linearized Euler equations to demonstrate the results obtained in Sections 5 and 6.1. In particular, we show the improvement of the convergence rates for the $\mathrm{gPC}$ coefficients produces by sGFVM due to the increased regularity of the solution statistics.

The linearized Euler equations are represented by the system of equations for the density $\rho$, velocity $u$ and pressure $p$ of an inviscid gas, which takes the form

$$
\frac{\partial \mathbf{U}}{\partial t}+\mathbf{A} \frac{\partial \mathbf{U}}{\partial x}=\mathbf{0}
$$

where

$$
\mathbf{U}=\left[\begin{array}{l}
\rho \\
u \\
p
\end{array}\right], \quad \mathbf{A}=\left[\begin{array}{ccc}
\bar{u} & \bar{\rho} & 0 \\
0 & \bar{u} & \frac{1}{\bar{\rho}} \\
0 & \gamma \bar{p} & \bar{u}
\end{array}\right]
$$

The matrix $\mathbf{A}$ has the eigenvalue decomposition $\mathbf{A}=\mathbf{R} \mathbf{\Lambda} \mathbf{R}^{-1}$ with

$$
\mathbf{\Lambda}=\operatorname{diag}(\bar{u}-\bar{a}, \bar{u}, \bar{u}+\bar{a}), \quad \mathbf{R}=\left[\begin{array}{ccc}
-\frac{\bar{\rho}}{\bar{a}} & 1 & \frac{\bar{\rho}}{\bar{a}} \\
1 & 0 & 1 \\
-\bar{\rho} \bar{a} & 0 & \bar{\rho} \bar{a}
\end{array}\right]
$$

where $\bar{a}=\sqrt{\frac{\gamma \bar{p}}{\bar{\rho}}}$ is the sound speed. We set $\gamma=7 / 5, \bar{\rho}=1, \bar{u}=1$ and $\bar{p}=1 / \gamma$.

Taking in (7.8) $\mathbf{F}(\mathbf{U})=\mathbf{A U}$ we get

$$
\sum_{k=0}^{N} \frac{\partial \mathbf{U}_{k}}{\partial t} M_{k i}+\int_{Y} \mathbf{A} \frac{\partial}{\partial x} \sum_{k=0}^{N} \mathbf{U}_{k} \varphi_{k}(y) \varphi_{i}(y) \rho(y) \mathrm{d} y=\mathbf{0},
$$

which is equivalent to

$$
\sum_{k=0}^{N} \frac{\partial \mathbf{U}_{k}}{\partial t} M_{k i}+\sum_{k=0}^{N} \mathbf{A} \frac{\partial \mathbf{U}_{k}}{\partial x} M_{k i}=\mathbf{0} .
$$

Hence, the sGFVM for linearized Euler equations consists of solving $(N+1)$ advection equations to determine the coefficients $\mathbf{U}_{k}(x, t), k=0, \ldots, N$ :

$$
\begin{aligned}
& \frac{\partial \mathbf{U}_{k}}{\partial t}+\mathbf{A} \frac{\partial \mathbf{U}_{k}}{\partial x}=\mathbf{0} \\
& \sum_{k=0}^{N} \mathbf{U}_{k}^{(0)} M_{k i}=\int_{Y} \mathbf{U}_{0}(x, y) \varphi_{i}(y) \rho(y) \mathrm{d} y .
\end{aligned}
$$

According to the Theorem 7.1, the gPC coefficients are smoother than the path-wise solution of the conservation law with Riemann initial data with uncertain shock location; in particular, the regularity of the coefficients is determined by the regularity of the given probability density function for the initial shock position.

Consider first the test case in which the initial condition is taken as a sine-wave with random initial phase:

$$
\mathbf{U}_{0}(x, y)=\left[\begin{array}{l}
\rho_{0} \\
u_{0} \\
p_{0}
\end{array}\right]=\left[\begin{array}{c}
\sin (2.0 \pi(x+0.1 y)) \\
\sin (2.0 \pi(x+0.1 y+0.1)) \\
\sin (2.0 \pi(x+0.1 y-0.1))
\end{array}\right],
$$

and the phase $y=Y(\omega)$ is distributed with probability density

$$
\mu(\xi)=\frac{1}{A} \mathrm{e}^{-\frac{1}{1-\xi^{2}}}
$$




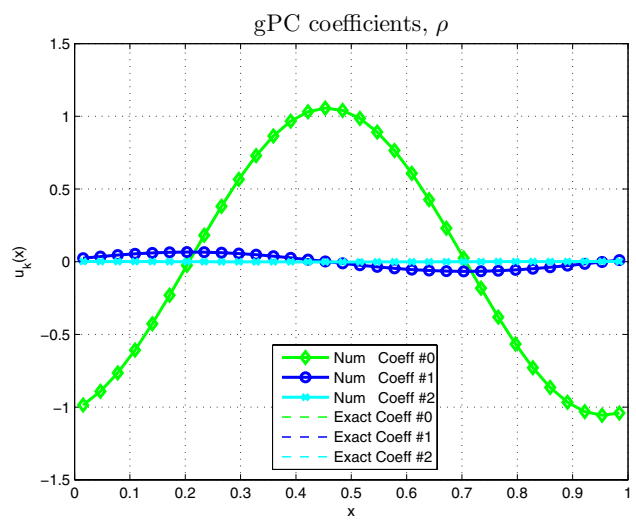

a) gPC coefficients

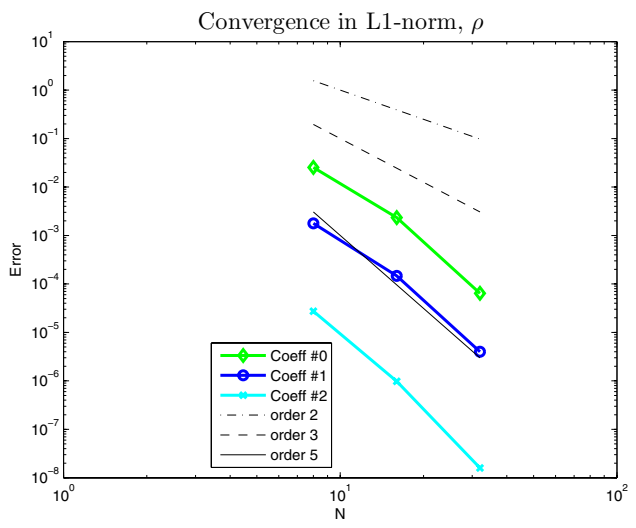

b) error vs resolution

FiguRE 6. Stochastic Galerkin method for linearized Euler equations: density.

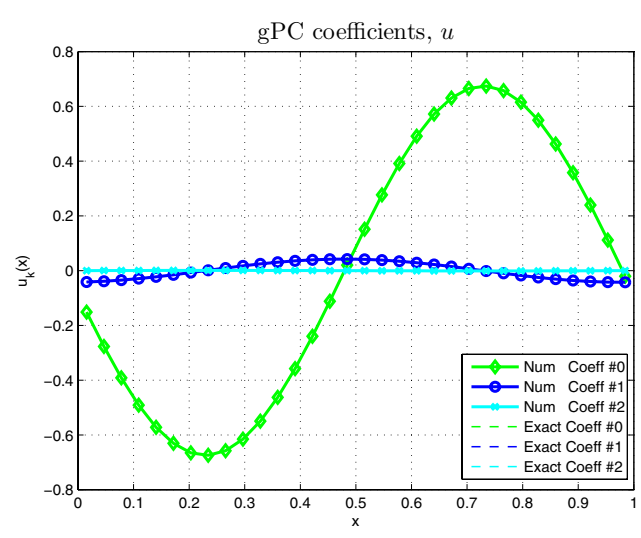

a) gPC coefficients

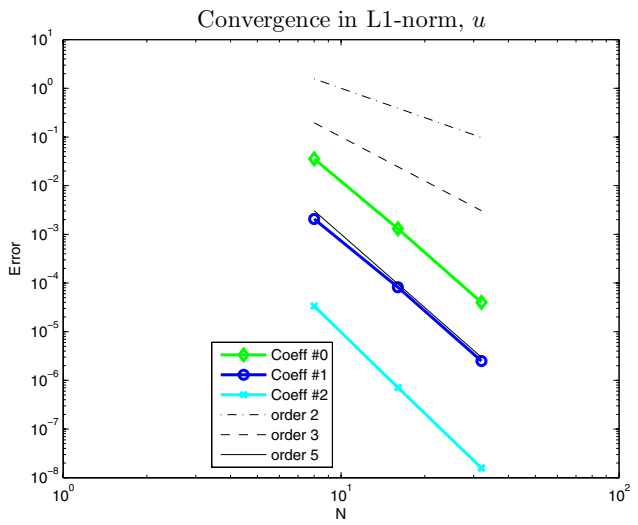

b) error vs resolution

FiguRE 7. Stochastic Galerkin method for linearized Euler equations: velocity.

where $A$ is the normalization constant. Note that $\mu \in C_{0}^{\infty}(-1,1)$. Therefore, under the theory developed in this paper, we can expect higher convergence rates in the direct FV computation of the gPC coefficients as compared to "pathwise" FV approximation of these quantities in Monte Carlo type methods.

The solution to (7.16)-(7.17) is computed using 5th order WENO scheme [25]; the gPC coefficients and convergence rates are illustrated in Figures 6-8. Note that for this type of the initial data we observe an optimal 5 th order of convergence, which is anticipated since the initial condition is a smooth function.

Next, to demonstrate the consequences of the obtained regularity results on high-order approximations of gPC coefficients we solve (7.16)-(7.17) with the following Riemann initial data:

$$
\mathbf{U}_{0}(x, y)=\left\{\begin{array}{l}
\mathbf{U}_{L}, x<y ; \\
\mathbf{U}_{R}, x>y,
\end{array}\right.
$$

where

$$
\mathbf{U}_{L}=\left[\begin{array}{l}
1.0 \\
0.0 \\
1.5
\end{array}\right], \quad \mathbf{U}_{R}=\left[\begin{array}{l}
0.1 \\
0.0 \\
0.2
\end{array}\right],
$$

$y=x_{0}+Y(\omega), x_{0}$ is a known constant and $Y(\omega)$ is distributed with the same probability density function (7.19). 


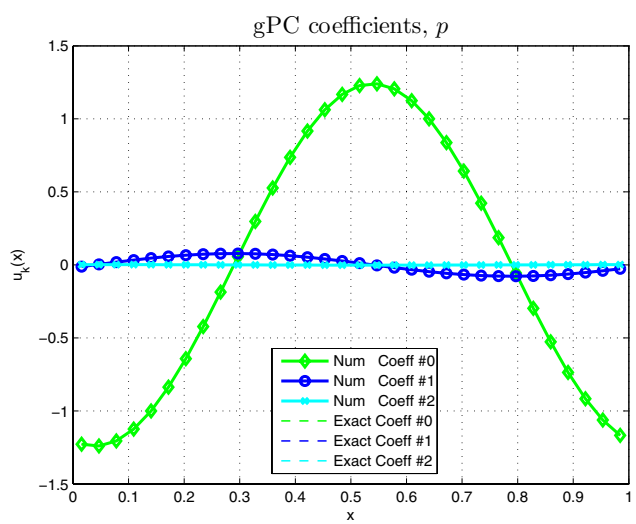

a) gPC coefficients

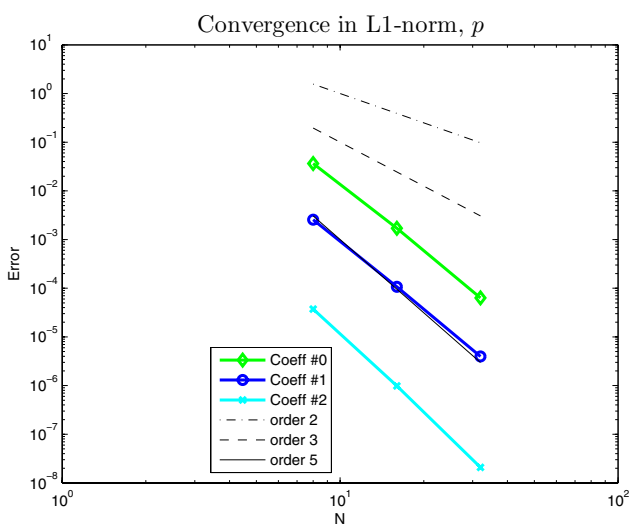

b) error vs resolution

FiguRE 8. Stochastic Galerkin method for linearized Euler equations: pressure.

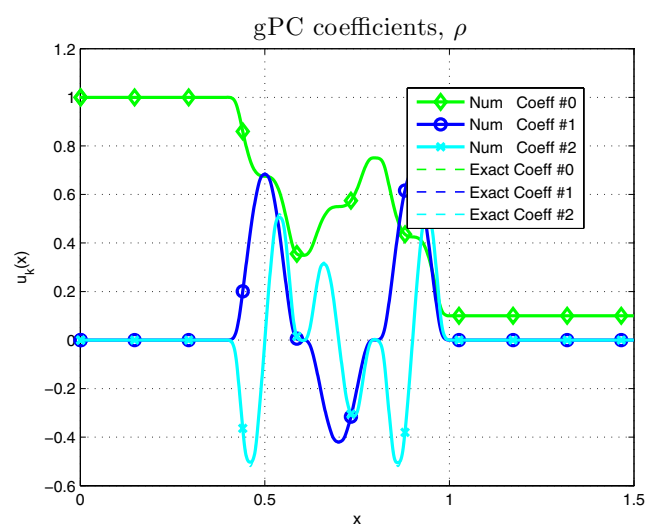

a) gPC coefficients

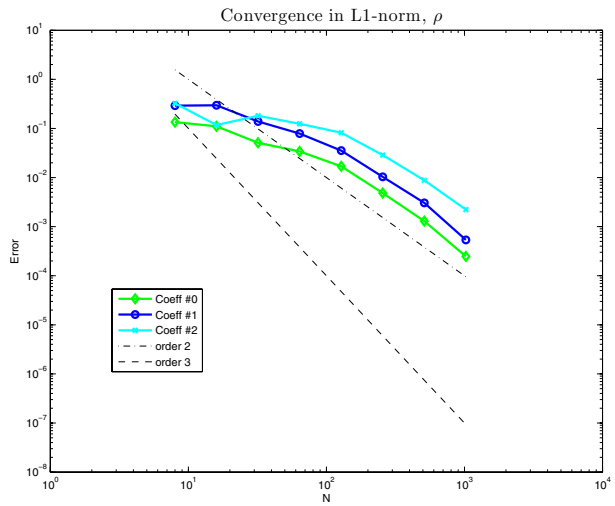

b) error vs resolution

FiguRE 9. Stochastic Galerkin method for linearized Euler equations: density.

Consider first the approximation of the gPC coefficients obtained using the sGFVM with three gPC terms. Figures 9a), 10a) and 11a) illustrate the polynomial coefficients for each of the variables $\rho, u$ and $p$, and the corresponding convergence plot is shown in Figures 9b), 10b) and 11b). In this computation, the 3rd order WENO scheme [25] was used to approximate the solution of (7.16)-(7.17). We note that the average convergence rate reaches the value of 3 , while the path-wise simulations of shock solutions would result in the first order of accuracy as a maximum. Figures 12-14 correspond to the sGFVM with six gPC terms based on 5th order WENO solver to the Galerkin systems. The results show the convergence rate reaching the value of 5 asymptotically.

Note that in Figures 9-14 one can observe the first-order convergence for small $N$ (number of cells in $x$ ) and high-order convergence for bigger $N$. The reason for that is the insufficient approximation of the polynomial coefficients of the gPC expansion on coarse grids which is improved by the refinement of the spacial mesh.

Hence, we have demonstrated how the smoothness of the gPC coefficients for problems with Riemann initial data and random discontinuity position affects the convergence rates of the sGFVM method, thus making the gPC based computations more efficient as compared to the methods based on the simulation of the pathwise shock solutions. 


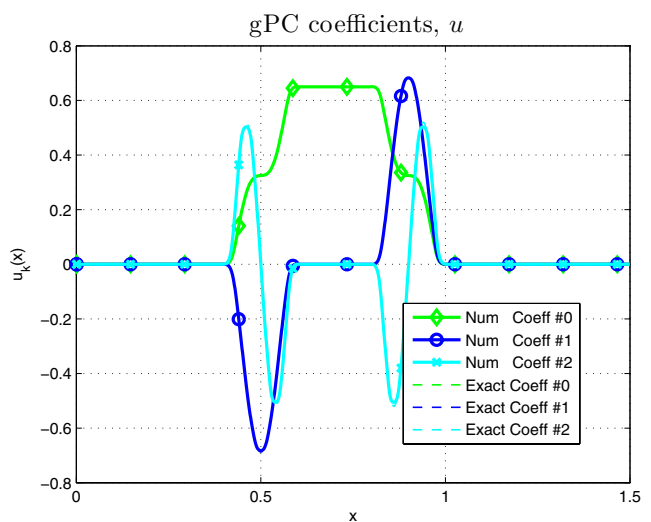

a) gPC coefficients

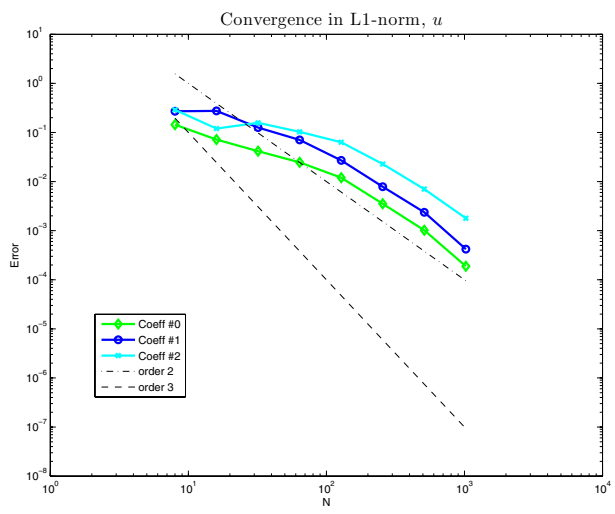

b) error vs resolution

Figure 10. Stochastic Galerkin method for linearized Euler equations: velocity.

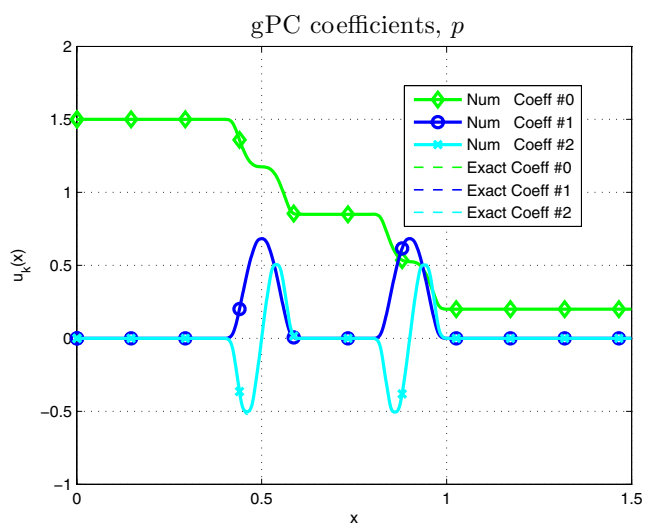

a) gPC coefficients

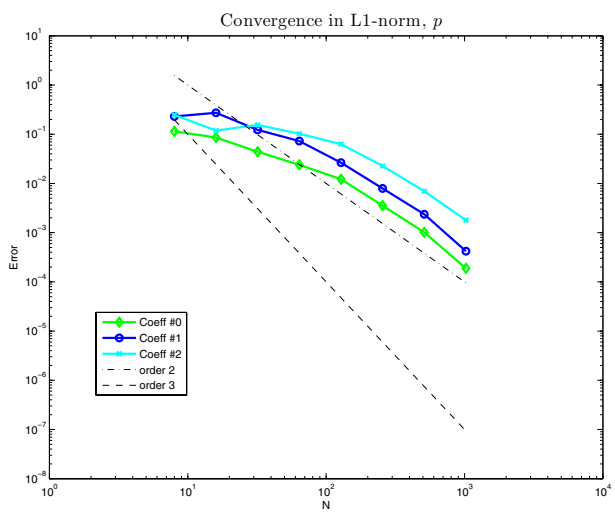

b) error vs resolution

FiguRE 11. Stochastic Galerkin method for linearized Euler equations: pressure.

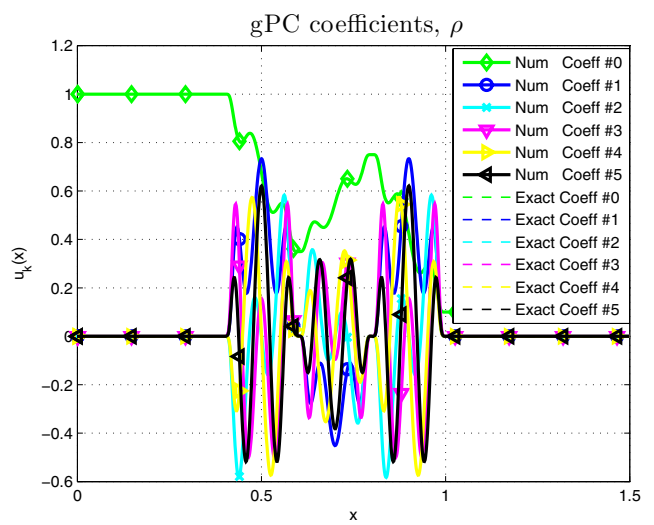

a) gPC coefficients

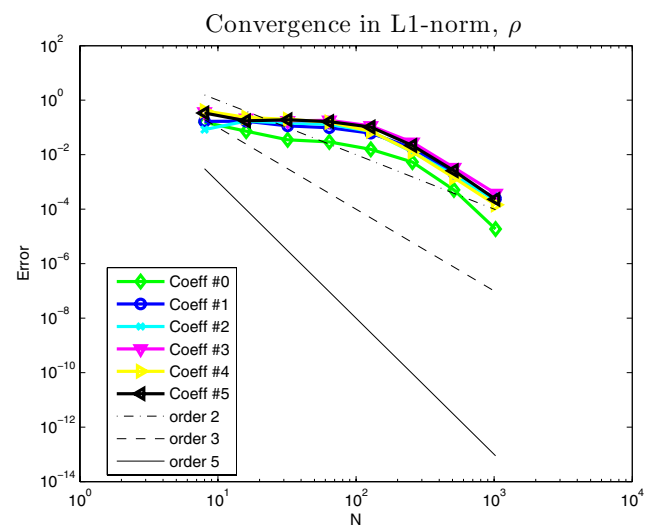

b) error vs resolution

FiguRE 12. Stochastic Galerkin method for linearized Euler equations: density. 


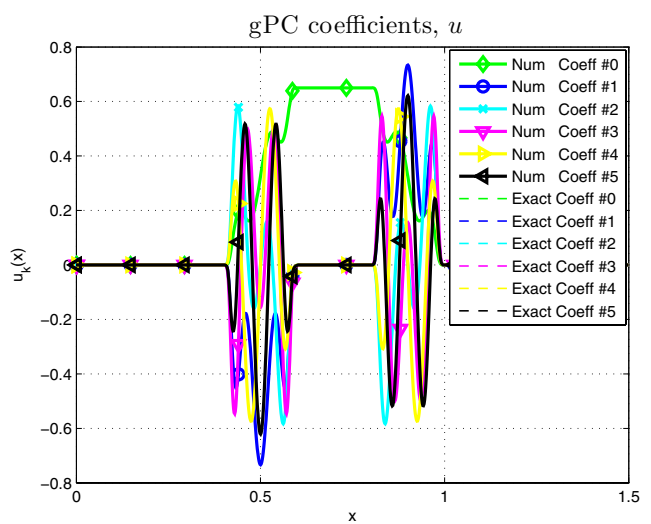

a) gPC coefficients

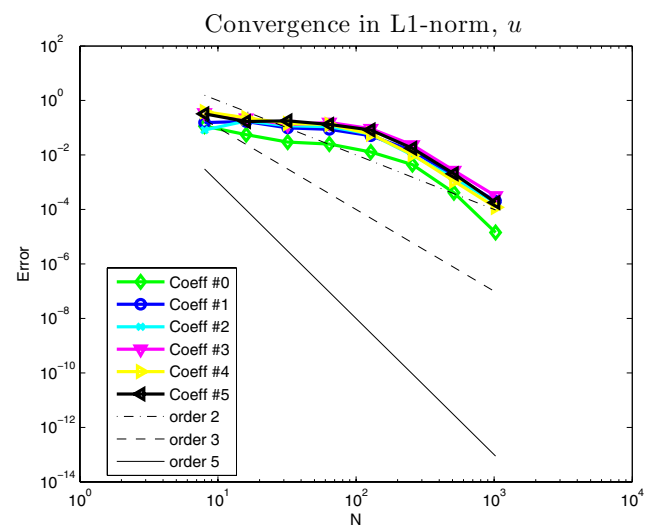

b) error vs resolution

FIgURE 13. Stochastic Galerkin method for linearized Euler equations: velocity.

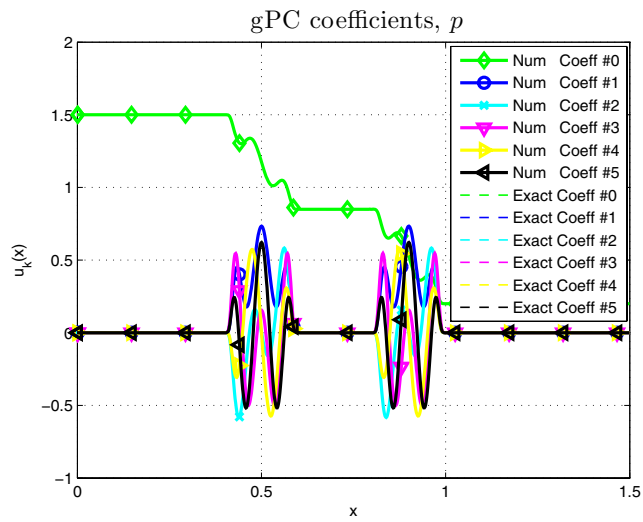

a) gPC coefficients

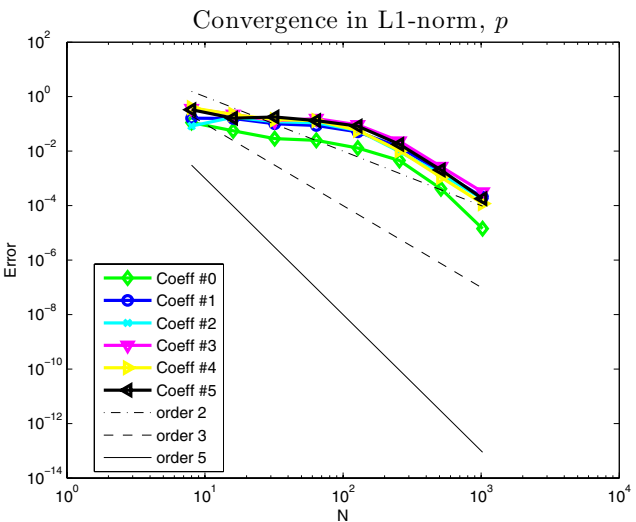

b) error vs resolution

FiguRE 14. Stochastic Galerkin method for linearized Euler equations: pressure.

\section{Conclusions}

We have investigated the regularity properties of random entropy solutions to hyperbolic conservation laws with random initial data for both scalar equations and certain hyperbolic systems in one space dimension. For Riemann initial data with random shock location we have proven that the resulting probabilistic shock profiles exhibit additional smoothness related to the probability density function of the distribution law of the shock location in the initial data. This smoothing effect is unrelated to viscosity and is found even in cases where the path-wise entropy solutions develop shocks. We have proven that this holds true also for statistical moments like the mean, variance, higher order spatio-temporal correlation functions as well as the coefficients in the gPC expansion. The improved regularity was shown numerically to imply absence of discontinuities in hyperbolic systems obtained by stochastic Galerkin projections onto truncated generalized polynomial chaos expansions of random entropy solutions. This opens the possibility for developing high-order convergent numerical schemes by combining intrusive gPC-type Galerkin discretizations in stochastic space with standard high-order finite volume discretizations in physical space. The generalization of the obtained results to multidimensional equations and systems of equations is subject of ongoing research and will be reported elsewhere. 


\section{REFERENCES}

[1] E. Godlewski and P. Raviart, Hyperbolic systems of conservation laws. Ellipses Publ., Paris (1995).

[2] R. LeVeque, Numerical methods for conservation laws. Birkhäuser Verlag (1992).

[3] S. Mishra and Ch. Schwab, Sparse tensor multi-level Monte Carlo finite volume methods for hyperbolic conservation laws with random intitial data. Math. Comput. 81 (2012) 1979-2018.

[4] S. Mishra, Ch. Schwab and J. Šukys, Multi-level Monte Carlo finite volume methods for nonlinear systems of conservation laws in multi-dimensions. J. Comput. Phys. 231 (2012) 3365-3388.

[5] S. Mishra, Ch. Schwab and S. Tokareva, Stochastic Finite Volume methods for uncertainty quantification in hyperbolic conservation laws. In preparation (2012).

[6] J. Troyen, O. Le Maître, M. Ndjinga and A. Ern, Intrusive Galerkin methods with upwinding for uncertain nonlinear hyperbolic systems. J. Comput. Phys. 229 (2010) 6485-6511.

[7] J. Troyen, O. Le Maître, M. Ndjinga and A. Ern, Roe solver with entropy corrector for uncertain hyperbolic systems. J. Comput. Phys. 235 (2010) 491-506.

[8] E. H. Lieb and M. Loss, Analysis: 2nd Ed. Amer. Math. Soc. Graduate Studies in Math. 14 (2001).

[9] O.G. Ernst, A. Mugler, H.J. Starkloff and E. Ullmann, On the convergence of generalized polynomial chaos expansions. ESAIM: M2AN 46 (2012) 317-339.

[10] D. Xiu and G.E. Karniadakis, Modeling uncertainty in steady state diffusion problems via generalized polynomial chaos. Comput. Methods Appl. Mech. Engrg. 191 (2002) 4927-4948.

[11] D. Xiu and G.E. Karniadakis, Modeling uncertainty in flow simulations via generalized polynomial chaos. J. Comput. Phys. 187 (2003) 137-167.

[12] R. Abgrall, A simple, flexible and generic deterministic approach to uncertainty quantification in non-linear problems. Rapport de Recherche, INRIA 00325315 (2007).

[13] G. Poëtte, B. Després and D. Lucor, Uncertainty quantification for systems of conservation laws. J. Comput. Phys. 228 (2009) 2443-2467.

[14] R. Ghanem and P. Spanos, Stochastic Finite Elements: A Spectral Approach. Dover (2003).

[15] D. Gottlieb and D. Xiu, Galerkin method for wave equations with uncertain coefficients. Commun. Comput. Phys. 3 (2008) $505-518$

[16] G. Lin, C.-H. Su and G.E. Karniadakis, Predicting shock dynamics in the presence of uncertainties. J. Comput. Phys. 217 (2006) 260-276.

[17] G. Lin, C.-H. Su and G.E. Karniadakis, Stochastic modelling of random roughness in shock scattering problems: theory and simulations. Comput. Methods Appl. Mech. Eng. 197 (2008) 3420-3434.

[18] X. Wan and G.E. Karniadakis, Multi-element generalized polynomial chaos for arbitrary probability measures. SIAM J. Sci. Comput. 28 (2006) 901-928.

[19] B. Debusschere, H. Najm, P. Pébay, O. Knio, R. Ghanem and O. Le Maître, Numerical challenges in the use of polynomial chaos representations for stochastic processes. SIAM J. Sci. Comput. 26 (2004) 698-719.

[20] O. Knio and O. Le Maître, Uncertainty propagation in CFD using polynomial chaos decomposition. Fluid. Dynam. Res. 38 (2006) 616-640.

[21] O. Le Maître, O. Knio, H. Najm and R. Ghanem, Uncertainty propagation using Wiener-Haar expansions. J. Comput. Phys. 197 (2004) $28-57$.

[22] O. Le Maître, H. Najm, R. Ghanem and O. Knio, Multi-resolution analysis of Wiener-type uncertainty propagation schemes. J. Comput. Phys. 197 (2004) 502-531.

[23] O. Le Maître, H. Najm, P. Pébay, R. Ghanem and O. Knio, Multi-resolution analysis scheme for uncertainty quantification in chemical systems. SIAM J. Sci. Comput. 29 (2007) 864-889.

[24] T. Barth, On the propagation of the statistical model parameter uncertainty in CFD calculations. Theoret. Comput. Fluid Dyn. 26 (2012) 435-457.

[25] C.W. Shu, High order ENO and WENO schemes for computational fluid dynamics. In High-Order Methods for Computational Phys. Springer 9 (1999). 